\title{
Fast-Acting Insulin Aspart: A Review of its Pharmacokinetic and Pharmacodynamic Properties and the Clinical Consequences
}

\author{
Hanne Haahr ${ }^{1}$. Tim Heise ${ }^{2}$ \\ Published online: 30 October 2019 \\ (c) The Author(s) 2019
}

\begin{abstract}
Fast-acting insulin aspart (faster aspart) is insulin aspart (IAsp) with two added excipients, L-arginine and niacinamide, to ensure formulation stability with accelerated initial absorption after subcutaneous administration compared with previously developed rapid-acting insulins. The pharmacokinetic/pharmacodynamic properties of faster aspart have been characterised in clinical pharmacology trials with comparable overall methodology. In subjects with type 1 (T1D) or type 2 (T2D) diabetes, the serum IAsp concentration-time and glucose-lowering effect profiles are left-shifted for faster aspart compared with IAsp. In addition, faster aspart provides earlier onset, doubling of initial exposure, and an up to 2.5 -fold increase in initial glucose-lowering effect within $30 \mathrm{~min}$ of subcutaneous injection, as well as earlier offset of exposure and effect. Similar results have been shown using continuous subcutaneous insulin infusion (CSII). The improved pharmacological properties of faster aspart versus IAsp are consistent across populations, i.e. in the elderly, children, adolescents and the Japanese. Thus, the faster aspart pharmacological characteristics more closely resemble the mealtime insulin secretion in healthy individuals, giving faster aspart the potential to further improve postprandial glucose control in subjects with diabetes. Indeed, change from baseline in 1-h postprandial glucose increment is in favour of faster aspart versus IAsp when used as basal-bolus or CSII treatment in phase III trials in subjects with T1D or T2D. This review summarises the currently published results from clinical pharmacology trials with faster aspart and discusses the potential clinical benefits of faster aspart compared with previous rapid-acting insulin products.
\end{abstract}

\section{Introduction}

In patients with diabetes, postprandial glucose (PPG) reduction constitutes an important aspect in optimising overall glycaemic control and reaching glycaemic targets $[1,2]$. In healthy individuals, insulin secretion occurs immediately after meal ingestion, thereby controlling PPG [3]. To address postprandial hyperglycaemia, the aspiration for patients with diabetes is a mealtime insulin with an absorption profile that mimics the endogenous postprandial insulin secretion in the healthy state [4-6].

Rapid-acting insulin is used in patients with type 1 diabetes (T1D) in basal-bolus treatment regimens or administered via continuous subcutaneous insulin infusion (CSII), and in patients with type 2 diabetes (T2D) who need to intensify

Hanne Haahr

hhaa@novonordisk.com

1 Clinical Pharmacology, Novo Nordisk A/S, Vandtårnsvej 114, 2860 Søborg, Denmark

2 Profil, Hellersbergstrasse 9, 41460 Neuss, Germany

\section{Key Points}

Despite the advantages of rapid-acting insulins over regular human insulin with respect to pharmacokinetic/ pharmacodynamic properties, there is still a need for accelerated insulin absorption and action to better mimic mealtime insulin secretion in the healthy state.

Faster aspart provides an overall left-shift of the pharmacokinetic/pharmacodynamic profiles resulting in earlier onset, twice as large initial exposure, and up to 2.5 -fold greater initial glucose-lowering effect within the first $30 \mathrm{~min}$, as well as earlier offset of exposure and effect compared with insulin aspart.

In phase III trials, the better resemblance of faster aspart pharmacological characteristics to healthy endogenous mealtime insulin secretion has been shown to lead to improved postprandial glycaemic control in subjects with diabetes relative to previously developed rapid-acting insulins. 
treatment by adding mealtime insulin to basal insulin plus oral antidiabetic drugs (OADs) [7]. Previously developed rapid-acting insulins (insulin aspart [IAsp], insulin lispro and insulin glulisine) provide faster absorption and earlier onset of glucose-lowering effect, leading to improved PPG control versus regular human insulin $[8,9]$. However, their absorption rates are insufficient to optimise postprandial glycaemia when insulin administration occurs at meal initiation $[10,11]$. Rather, the greatest PPG reduction is obtained when administering these insulin products $15-30$ min before a meal $[10,11]$. In line with approved labelling, and presumably for simplicity and practical reasons, many patients with diabetes employ only a limited or no interval between insulin administration and meal initiation [12]. Thus, there is a clinical need for mealtime insulins with ultra-fast absorption properties to further minimise the gap relative to mealrelated insulin secretion in the healthy state.

Fast-acting IAsp (faster aspart) is IAsp in a new formulation developed to achieve accelerated initial absorption after subcutaneous administration compared with previously developed rapid-acting insulins [13-15]. The

pharmacological properties of faster aspart have been characterised in several clinical pharmacology trials [16-27]. Moreover, phase III trials have investigated the efficacy and safety of faster aspart versus IAsp in subjects with T1D or T2D [28-35]. This review summarises the results from clinical pharmacology trials with faster aspart and relates these findings to the clinical benefits associated with faster aspart compared with IAsp based on outcomes from the phase III trials.

\section{Faster Aspart}

Faster aspart is a new formulation of IAsp (NovoRapid ${ }^{\circledR} /$ NovoLog ${ }^{\circledR}$ ), an analogue of human insulin where the proline in position B28 has been substituted with aspartate. Compared with IAsp, faster aspart was modified by adding two excipients-niacinamide (vitamin $\mathrm{B}_{3}$ ) to increase the absorption rate following subcutaneous administration, and L-arginine (an amino acid) to ensure formulation stability. In the original IAsp formulation, most IAsp molecules exist as hexamers, which are too large to be easily absorbed [13]. Under conditions simulating the pharmaceutical formulation or the subcutaneous depot, niacinamide increases the fraction of the more readily absorbable IAsp monomers, thereby partly eliminating the absorption rate-limiting step of hexamer dissociation into monomers [13]. Accordingly, niacinamide promotes the trans-endothelial transport of IAsp [13]. Furthermore, studies in pigs indicate that niacinamide may increase skin blood flow via transient, local vasodilatation, which could also promote absorption of IAsp molecules after subcutaneous administration [13].

Niacinamide and L-arginine are both included in the US FDA database of inactive ingredients in products for injection at higher concentrations than those occurring in faster aspart, and in the generally recognised as safe (GRAS) food substance database [36, 37]. Over several decades, studies with pharmacological doses of oral niacinamide in humans have shown a good safety profile [38]. Any local adverse effects of niacinamide at the injection site are unlikely since the half-life of niacinamide disappearance from the subcutaneous depot in pigs was shown to be only $\sim 5 \mathrm{~min}$ [13]. The amino acid L-arginine occurs naturally in protein-rich food, and supplementation up to 5-10 times the average daily dietary intake is safe and well tolerated [39].

Before choosing the faster aspart formulation intended for further clinical development, systematic pharmacokinetic assessment of various formulations was undertaken in humans to reach an informed decision on the optimal formulation, while balancing absorption rate and formulation stability [27]. Niacinamide positively affects the absorption rate, partly through increased monomerization [13], which, however, also adversely affects formulation stability. In contrast, another excipient, zinc, increases oligomerization of insulin, and therefore negatively impacts the absorption rate, but is important in ensuring formulation stability [40]. Figure 1 shows a conceptual model based on clinical pharmacokinetic data describing how the rate of absorption depends on varying concentrations of niacinamide and zinc in the faster aspart formulation. Several faster aspart formulations with different combinations of niacinamide and zinc concentrations were tested in a clinical pharmacology trial, and,

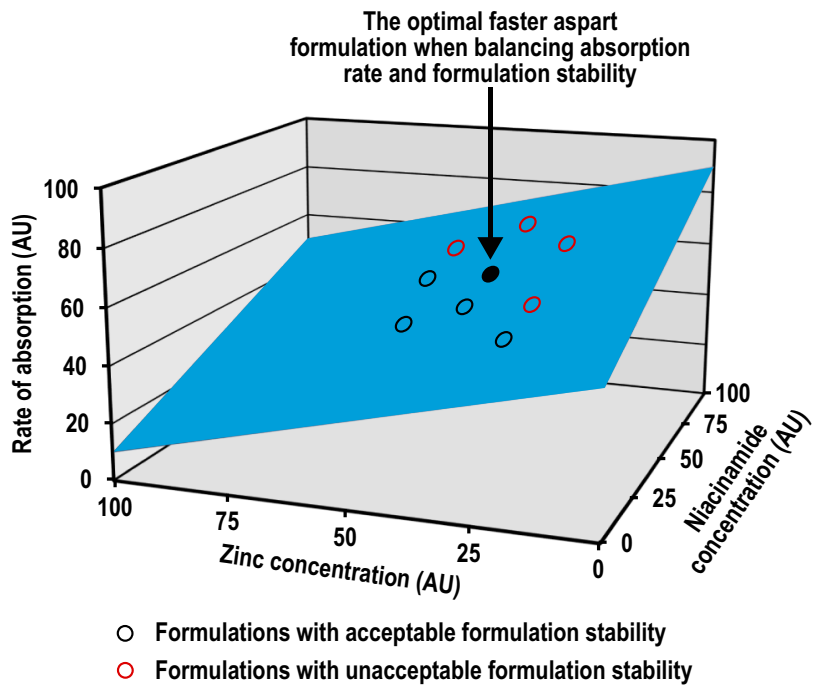

Fig. 1 Conceptual model showing the effect of varying zinc and niacinamide concentrations in the faster aspart formulation on the rate of absorption after subcutaneous administration. $A U$ arbitrary units 
among those with acceptable formulation stability, the one with the fastest absorption rate was chosen for further development. Recent investigations suggest that L-arginine also contributes to improved insulin stability through reduced insulin aggregation at high ionic strength [41].

\section{Methodology}

This review includes 12 clinical pharmacology trials [16-27] and 10 other clinical trials (most of them part of the Onset phase III clinical development programme) [28-35, 42, 43] with faster aspart, identified by PubMed searches for the terms 'faster aspart clinical trial' and 'faster acting insulin aspart onset', as well as a ClinicalTrials.gov search for phase I trials using the term 'faster aspart'. To be included in this review, it was required that trials had been published in article form, however with two exceptions, where inclusion was assessed highly relevant for the completeness of the review [27, 33].

Across the trials characterising the faster aspart pharmacological properties, the overall design and methodology were standardised as much as possible, while allowing minor variations depending on the specific trial population and objectives. To ensure consistency regarding experimental procedures and data analysis, all trials were single-centre trials and few sites were involved throughout the faster aspart clinical pharmacology programme. All trials were singledose trials, and IAsp was consistently included as a comparator, where relevant.

\subsection{Pharmacokinetics}

Blood samples were collected immediately before dosing and at frequent predefined time points until $12 \mathrm{~h}$ after dosing in most trials to capture the full duration of exposure in all subjects. In all trials, free serum IAsp concentrations were measured using a validated IAsp-specific enzyme-linked immunosorbent assay following polyethylene glycol (PEG) precipitation. In selected trials, total serum IAsp concentrations were measured using the same IAsp assay without PEG precipitation [21].

\subsection{Pharmacodynamics}

In most faster aspart clinical pharmacology trials, the glucose-lowering effect was evaluated in a euglycaemic glucose clamp using either ClampArt (Profil, Neuss, Germany) [19, 20, 24], Biostator (MTB Medizintechnik, Amstetten, Germany) [18], STG-22 glucose-controlled insulin infusion system (Artificial Endocrine Pancreas; NIKKISO Co. Ltd., Tokyo, Japan) [25] or a manual clamp [17]. To avoid interference from endogenous insulin on the glucose clamp results, subjects with T1D were included in all clamp trials, except one specifically conducted to investigate the pharmacological properties of faster aspart in subjects with T2D [17]. To minimise endogenous insulin secretion, the clamp blood glucose (BG) target level was lower in subjects with T2D $(5.0 \mathrm{mmol} / \mathrm{L})[17]$ than in those with T1D $(5.5 \mathrm{mmol} / \mathrm{L})$ [18-20, 24, 25]. Still, as with all other clamp studies in subjects with T2D, there is a risk of endogenous insulin secretion, which may complicate the interpretation of the study results [44]. In two clinical pharmacology trials [22, 23] and in several phase III trials [28-33], a standardised 4-6 h meal test was conducted to assess PPG excursion with faster aspart versus IAsp. In all trials to assess the pharmacodynamics of faster aspart, the subject's usual insulin was replaced and all insulin was terminated in due time to avoid any interference from exogenous insulin on the pharmacodynamic results.

\section{Pharmacokinetics of Faster Aspart}

The pharmacokinetics of faster aspart versus IAsp after subcutaneous injection were investigated in a pooled analysis of 218 adults with T1D based on six clinical pharmacology trials [16] and in 61 subjects with T2D based on one clinical pharmacology trial [17]. The pharmacokinetic profile was shifted to the left with faster aspart versus IAsp consistently across subjects with T1D or T2D (Fig. 2), suggesting that the faster aspart pharmacokinetic profile more closely mimics healthy endogenous insulin secretion relative to previously developed rapid-acting insulins.

\subsection{Onset and Early Exposure}

In the pooled analysis of adults with T1D, onset of appearance occurred $\sim 5$ min earlier, time to $50 \%$ of maximum concentration in the early part of the pharmacokinetic profile ( $t_{\text {Early }} 50 \%$ Cmax $)$ was $~ 10$ min shorter, and time to maximum concentration $\left(t_{\max }\right)$ was 7 min shorter for faster aspart versus IAsp (Fig. 3a) [16]. Throughout the faster aspart clinical pharmacology trials, onset of appearance, $t_{\text {Early }} 50 \%$ Cmax and $t_{\max }$ were used to assess the onset of exposure. Onset of appearance was defined as the time from dosing until the first serum IAsp concentration $\geq 10 \mathrm{pmol} / \mathrm{L}$ (the assay lower limit of quantification) and best reflects the true onset, whereas $t_{\text {Early } 50 \% ~ C \max }$ and $t_{\max }$ represent a composite of time to onset and rate of absorption [45]. Nevertheless, $t_{\text {Early } 50 \% C \max }$ and $t_{\max }$ were also derived for faster aspart to bridge to previous trials with rapid-acting insulin reporting one or both of these endpoints [46-49].

In line with the left-shifted pharmacokinetic profile, greater early exposure was seen for faster aspart versus IAsp within the first $2 \mathrm{~h}$ after administration in the pooled analysis of adults with T1D (Fig. 3b) [16]. From the time of dose 


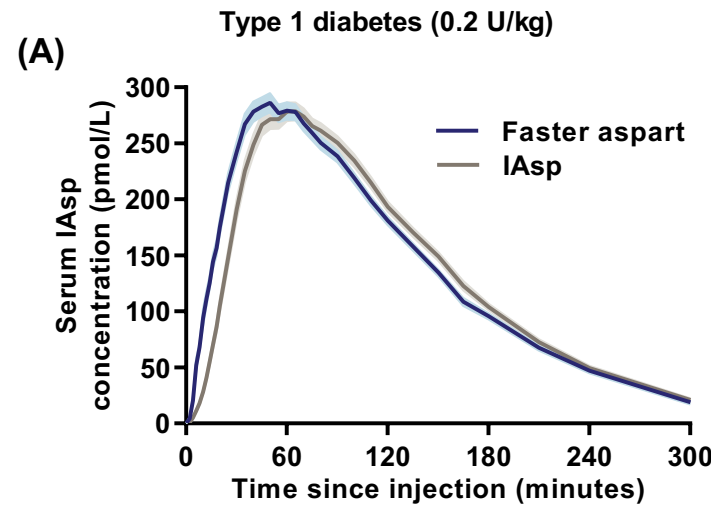

(C)

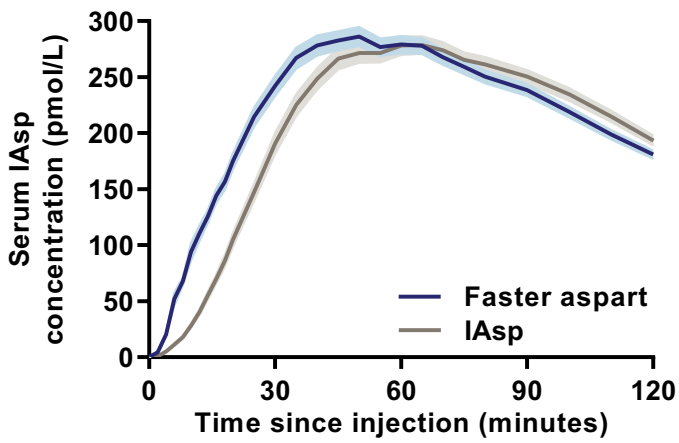

Fig. 2 Pharmacokinetic profiles for faster aspart versus IAsp in subjects with T1D and T2D. Mean 5-h (a), 6-h (b) or 2-h (c, d) serum IAsp concentration-time profiles after a subcutaneous dose of $0.2 \mathrm{U} /$ $\mathrm{kg}$ in T1D (a, c) and $0.3 \mathrm{U} / \mathrm{kg}$ in T2D (b, d). Variability bands show the standard error of the mean. IAsp insulin aspart, TID type 1 dia-

administration until $30 \mathrm{~min}$, faster aspart exposure was twice as large as IAsp exposure.

In subjects with T2D, earlier onset and greater early exposure were also shown with faster aspart versus IAsp [17]. Onset of appearance occurred 1.2 min earlier, $t_{\text {Early } 50 \% ~ C \max }$ was $8.5 \mathrm{~min}$ shorter and early exposure within the first 30 min after dosing $\left(\mathrm{AUC}_{\mathrm{IAsp}, 0-30 \mathrm{~min}}\right)$ was $89 \%$ larger for faster aspart versus IAsp (Fig. 3). Hence, the accelerated absorption of faster aspart versus IAsp occurs in both T1D and T2D.

\subsection{Offset and Late Exposure}

Approaching healthy postprandial insulin secretion implies not only earlier onset and faster absorption rate but also earlier offset and lower late-phase exposure in order to reduce the risk of late postprandial hypoglycaemia [5]. In the pooled analysis of adults with T1D, offset of exposure occurred earlier for faster aspart than for IAsp. The time to $50 \%$ of maximum IAsp concentration in the late part of the pharmacokinetic profile $\left(t_{\text {Late } 50 \% \text { Cmax }}\right)$ was 12.2 min shorter $(p<0.001)$ and late exposure from $2 \mathrm{~h}$ onwards $\left(\mathrm{AUC}_{\text {IAsp, } 2-\mathrm{t}}\right)$ was $11 \%$ smaller $(p<0.001)$ for faster aspart versus IAsp

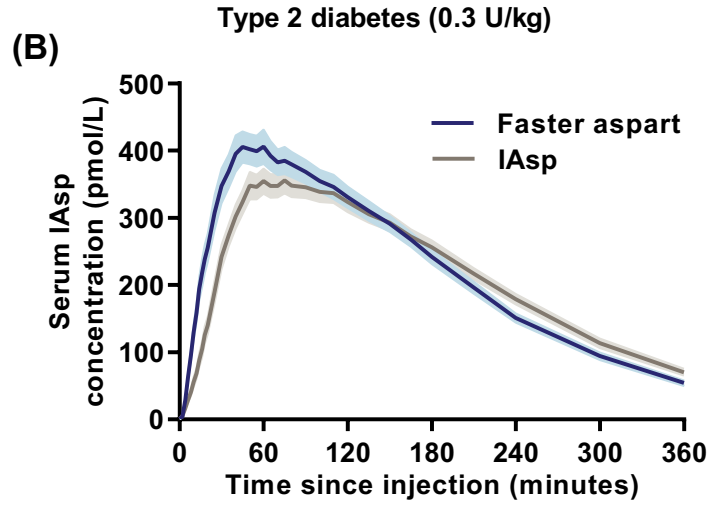

(D)

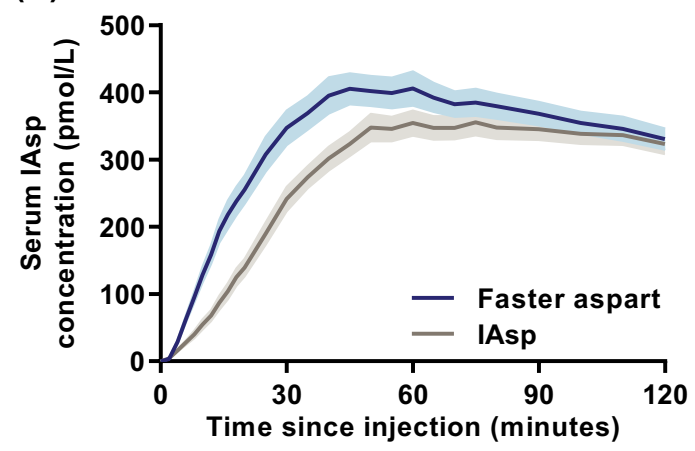

betes, $T 2 D$ type 2 diabetes, $U$ units. Modified from Heise et al. [16] under the terms of the Creative Commons Attribution-NonCommercial 4.0 International License (http://creativecommons.org/licenses/ by-nc/4.0/) and from Pieber et al. [17]

[16]. In subjects with T2D, the corresponding differences for offset and late exposure were $36.4 \mathrm{~min}(p<0.001)$ and $12 \%$ $(p=0.002)$, respectively, both in favour of faster aspart [17]. Thus, the better resemblance of the faster aspart pharmacokinetic profile to postprandial endogenous insulin secretion in healthy individuals pertains to both the early and late parts of the profile.

\subsection{Overall Exposure}

In the pooled analysis of adults with $\mathrm{T} 1 \mathrm{D}$, total exposure $\left(\mathrm{AUC}_{\mathrm{IAsp}, 0-\mathrm{t}}\right)$ and maximum concentration $\left(C_{\max }\right)$ did not differ between faster aspart and IAsp. The treatment ratios of faster aspart/IAsp were 1.01 (95\% confidence interval [CI] $0.98-1.04, p=0.470)$ and 1.04 (95\% CI 1.00-1.08, $p=0.085)$, respectively [16]. In subjects with T2D, $\mathrm{AUC}_{\mathrm{IAsp}, 0-\mathrm{t}}$ was similar for faster aspart and IAsp, with a treatment ratio of 0.99 (95\% CI 0.94-1.04, $p=0.646$ ), while $C_{\max }$ was slightly higher for faster aspart than for IAsp, with a treatment ratio of 1.13 (95\% CI 1.02-1.24, $p=0.018$ ) [17]. It is important for the interpretation of $t_{\text {Early } 50 \% ~ C \max }$ and $t_{\text {Late } 50 \% \text { Cmax }}$ that $\mathrm{C}_{\max }$ is comparable between faster aspart and IAsp [45]. The higher $C_{\max }$ for faster aspart versus IAsp 
Fig. 3 Onset of exposure (a) and early exposure (b) for faster aspart versus IAsp after a subcutaneous dose of $0.2 \mathrm{U} / \mathrm{kg}$ in subjects with T1D and $0.3 \mathrm{U} /$ $\mathrm{kg}$ in subjects with T2D. ${ }^{\mathrm{a}}$ Faster aspart-IAsp. ${ }^{b}$ For treatment comparison of faster aspart versus IAsp. ${ }^{c}$ Faster aspart/IAsp. $A U C$ area under the curve, $C I$ confidence interval, IAsp insulin aspart, $L S$ Mean least square mean, $T 1 D$ type 1 diabetes, $T 2 D$ type 2 diabetes, $t_{\text {Early }} 50 \%$ Cmax time to $50 \%$ of maximum concentration in the early part of the pharmacokinetic profile, $t_{\max }$ time to maximum concentration, $U$ units. Data from Heise et al. [16] and Pieber et al. [17]

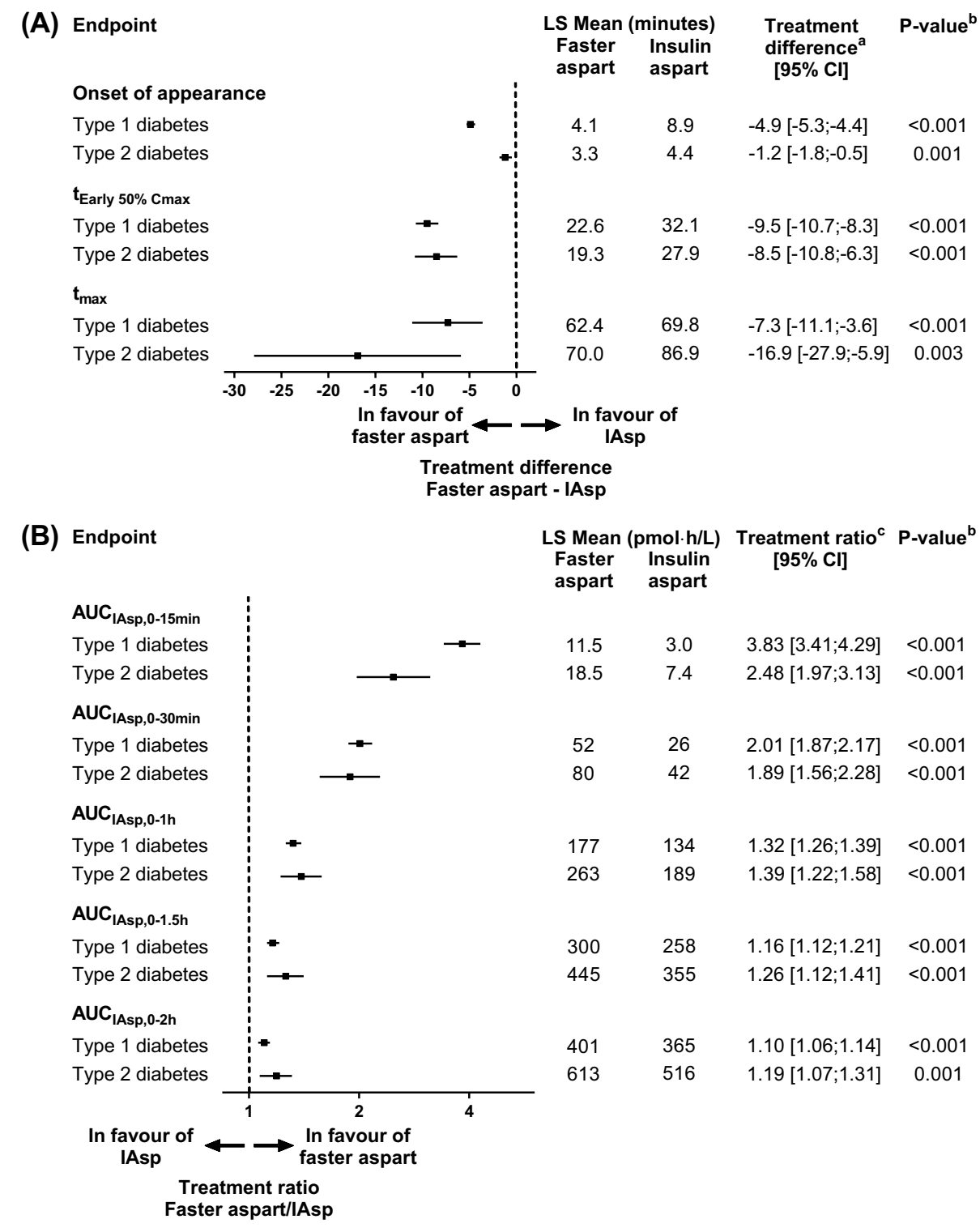

in subjects with T2D implies that both $t_{\text {Early } 50 \% C \max }$ and $t_{\text {Late }} 50 \%$ Cmax were artificially increased for faster aspart. If $C_{\text {max }}$ had been similar for faster aspart and IAsp, the treatment differences for $t_{\text {Early } 50 \% C \max }$ and $t_{\text {Late } 50 \% \mathrm{Cmax}}$ in favour of faster aspart would probably have been even larger [17].

Absolute bioavailability of faster aspart was determined in healthy males receiving faster aspart subcutaneously in the abdomen, upper arm and thigh, and intravenously [26]. Absolute bioavailability of faster aspart was $~ 80 \%$ independent of injection region (abdomen 83\%; upper arm 77\%; thigh $77 \%$ ) [26].

The dose-concentration relationship for faster aspart was investigated in subjects with T1D across a dose range of $0.1-0.4 \mathrm{U} / \mathrm{kg}$ [19]. Analysis of dose proportionality indicated that increases in $\mathrm{AUC}_{\mathrm{IAsp}, 0-\mathrm{t}}$ and $C_{\text {max }}$ with increasing dose were modestly larger than dose proportional and suggested a $12 \%$ increase in total exposure and $C_{\text {max }}$ following a $10 \%$ increase in faster aspart dose [19]. Comparable results were seen for IAsp, and it was concluded that this minor deviation from dose proportionality was not expected to influence dose titration in the clinical setting [19].

\subsection{Pharmacokinetics Measured as Free or Total Insulin Aspart (IAsp)}

Insulin can occur in the circulation as bound or free. The bound form occurs because of reversible binding to anti-insulin antibodies and is therefore primarily relevant to consider in previously insulin-treated individuals [50, 51]. Insulin pharmacokinetic assessment in subjects with diabetes must account for the potential assay interference coming from the presence of anti-insulin antibodies 
[51]. In all the faster aspart clinical pharmacology trials, free IAsp was measured following removal of anti-insulin antibodies by PEG precipitation. Because it adds to the totality of data, and on request by regulatory bodies, total IAsp was also measured in selected trials [18, 22, $24,25]$. Subsequently, a pooled analysis of four clinical pharmacology trials in adults with T1D compared the pharmacokinetics of faster aspart and IAsp based on total and free IAsp measurements. Earlier onset, greater early exposure and faster offset of faster aspart versus IAsp were shown regardless of being based on free or total IAsp [21]. The faster aspart-IAsp difference in $t_{\text {Early } 50 \% C \max }$ was $-8.8 \mathrm{~min}$ for free and $-7.6 \mathrm{~min}$ for total IAsp (both $p<0.001$ ). $\mathrm{AUC}_{\text {IAsp }, 0-30 \mathrm{~min}}$ was $88 \%$ and $77 \%$ greater for faster aspart versus IAsp when measured as free and total IAsp, respectively (both $p<0.001$ ). Finally, the faster aspart-IAsp difference in $t_{\text {Late }} 50 \%$ Cmax was $-13.8 \mathrm{~min}$ for free and $-14.0 \mathrm{~min}$ for total IAsp (both $p<0.001$ ) [21].

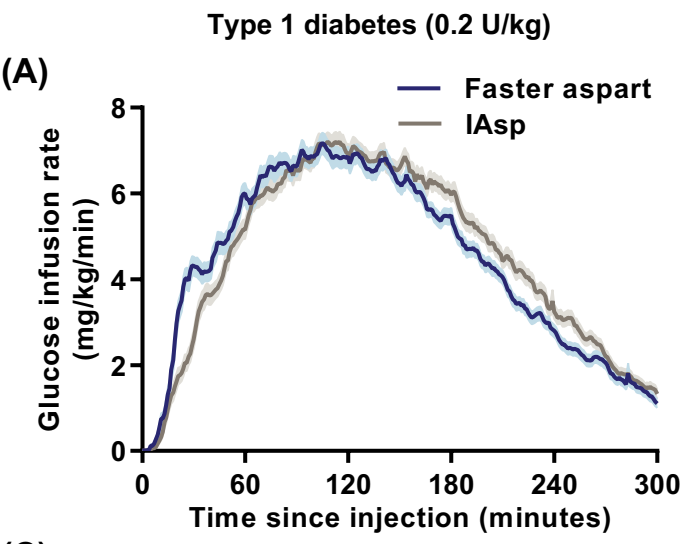

(C)

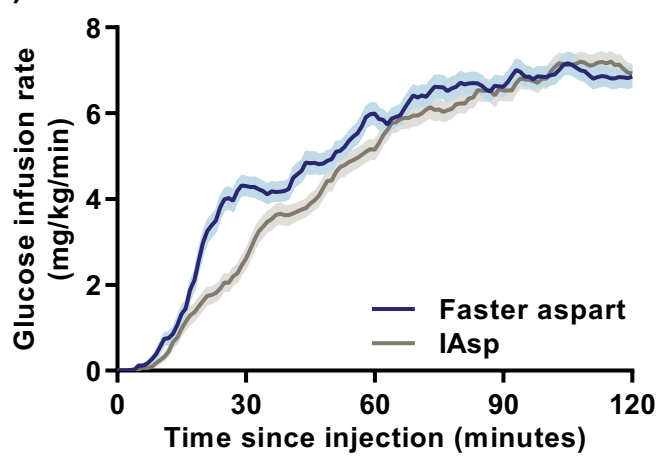

Fig. 4 Pharmacodynamic profiles for faster aspart versus IAsp in subjects with T1D and T2D. Mean 5-h (a), 6-h (b) or 2-h (c, d) glucoselowering effect profiles after a subcutaneous dose of $0.2 \mathrm{U} / \mathrm{kg}$ in T1D (a, c) and $0.3 \mathrm{U} / \mathrm{kg}$ in T2D (b, d). Variability bands show the standard error of the mean. IAsp insulin aspart, TID type 1 diabetes, T2D type

\section{Pharmacodynamics of Faster Aspart}

The pharmacodynamics of faster aspart versus IAsp after subcutaneous injection were characterised in a pooled analysis of three glucose clamp trials including 119 adults with T1D [16] and in a glucose clamp trial including 61 subjects with T2D [17]. In accordance with the pharmacokinetic profile, the glucose-lowering effect profile was left-shifted for faster aspart versus IAsp in subjects with both T1D and T2D (Fig. 4).

\subsection{Onset and Early Glucose-Lowering Effect}

In the pooled analysis of adults with T1D, onset of action occurred $\sim 5$ min earlier, time to $50 \%$ of maximum glucose infusion rate (GIR) in the early part of the GIR profile $\left(t_{\text {Early }} 50 \%\right.$ GIRmax $)$ was 9.5 min shorter and time to maximum GIR $\left(t_{\mathrm{GIRmax}}\right)$ was 10.5 min shorter for faster aspart versus IAsp (Fig. 5a) [16]. Onset of action was used as the best possible estimation of the difference in onset of glucose-lowering effect between two insulins. Onset of

(B)

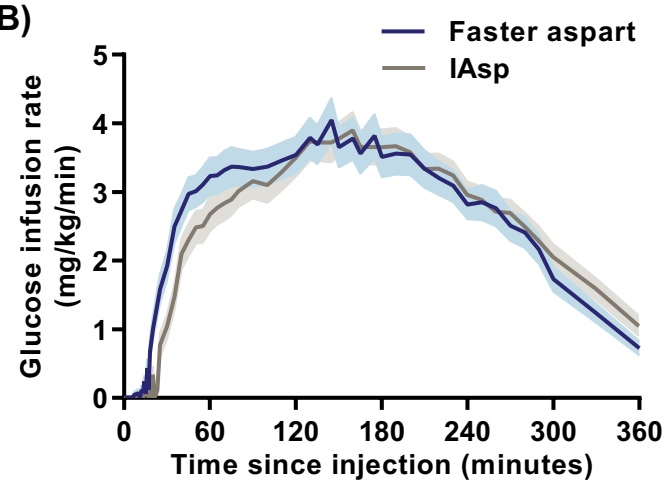

(D)

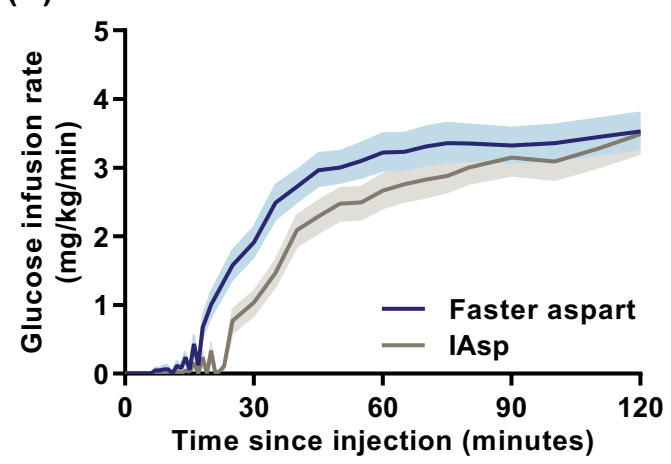

2 diabetes, $U$ units. Modified from Heise et al. [16] under the terms of the Creative Commons Attribution-NonCommercial 4.0 International License (http://creativecommons.org/licenses/by-nc/4.0/) and from Pieber et al. [17] 
Fig. 5 Onset of glucoselowering effect (a) and early glucose-lowering effect (b) for faster aspart versus IAsp after a subcutaneous dose of $0.2 \mathrm{U} / \mathrm{kg}$ in subjects with T1D and $0.3 \mathrm{U} / \mathrm{kg}$ in subjects with T2D.

${ }^{\mathrm{a}}$ Faster aspart-IAsp. ${ }^{\mathrm{b}}$ For treatment comparison of faster aspart versus IAsp. ${ }^{c}$ Faster aspart/IAsp. AUC area under the curve, $C I$ confidence interval, GIR glucose infusion rate, IAsp insulin aspart, LS Mean least square mean, $T 1 D$ type 1 diabetes, $T 2 D$ type 2 diabetes, $t_{\text {Early } 50 \% \text { GIRmax }}$ time to $50 \%$ of maximum glucose infusion rate in the early part of the glucose infusion rate profile, $t_{\text {GIRmax }}$ time to maximum glucose infusion rate, $U$ units. Data are from Heise et al. [16] and Pieber et al. [17]

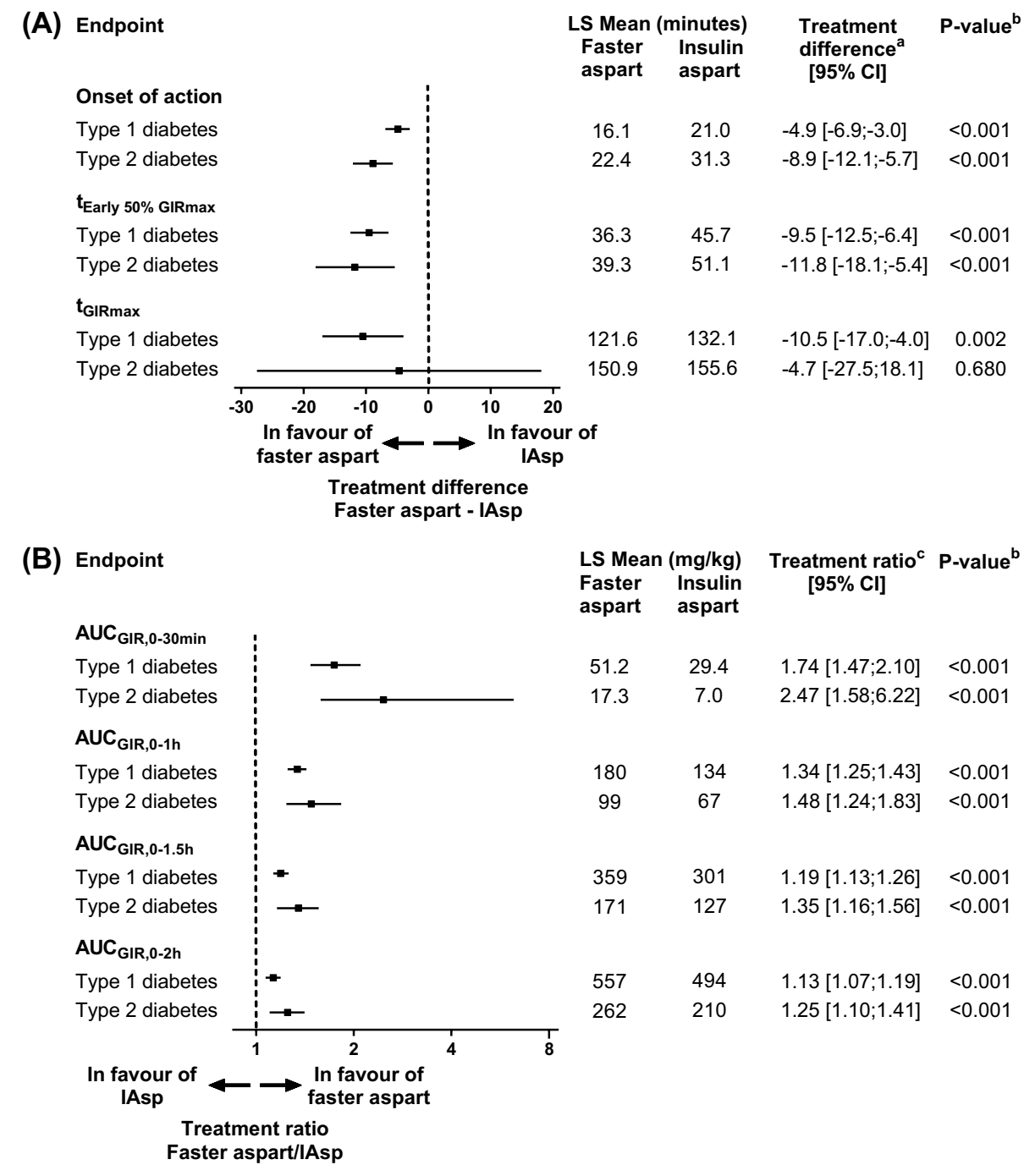

action was defined as the time from dosing until BG had decreased $\geq 0.3 \mathrm{mmol} / \mathrm{L}$ from baseline in a glucose clamp setting, where no glucose was infused from $60 \mathrm{~min}$ before dosing until the time of onset of action [19]. This definition includes the initial rate of action and therefore may overestimate the time to onset of action [45]. Furthermore, a glucose clamp is an artificial setting and therefore onset of action determined in a glucose clamp may not represent the true onset of action. Nevertheless, the current definition and derivation is relatively robust and clinically relevant, and gives a better estimate of first insulin action than provided by alternative glucose clamp-based endpoints such as time to reach $10 \%$ or $50 \%$ of maximum glucose-lowering effect or time to achieve $10 \%$ of total glucose-lowering effect [ 45 , $46,52,53]$.

In accordance with the left-shifted GIR profile for faster aspart versus IAsp, greater early glucose-lowering effect was observed for faster aspart up to $2 \mathrm{~h}$ after dosing in the pooled analysis of adults with T1D (Fig. 5b) [16]. Within the first
30 min after dosing, the glucose-lowering effect was $74 \%$ greater for faster aspart versus IAsp.

Earlier onset and greater early glucose-lowering effect were also shown for faster aspart versus IAsp in subjects with T2D [17]. Onset of action occurred 8.9 min earlier, $t_{\text {Early } 50 \% \text { GIRmax }}$ was 11.8 min shorter and early glucose-lowering effect up to $30 \mathrm{~min}$ after dosing $\left(\mathrm{AUC}_{\mathrm{GIR}, 0-30 \mathrm{~min}}\right)$ was $147 \%$ larger for faster aspart versus IAsp (Fig. 5).

\subsection{Offset and Late Glucose-Lowering Effect}

End of action has previously been derived as the time when GIR is no longer necessary and BG has increased to $8.3 \mathrm{mmol} / \mathrm{L}$ at the end of a glucose clamp [44, 54]. However, this definition overestimates end of action and may not be the most clinically relevant measure for mealtime insulins, where timely disposal of the glucose load originating from a meal is the main focus rather than continuously maintaining euglycaemia. Therefore, in the faster aspart 
clinical pharmacology trials, time to $50 \%$ of maximum GIR in the late part of the GIR profile ( $t_{\text {Late }} 50 \%$ GIRmax $)$ and late glucose-lowering effect from $2 \mathrm{~h}$ onwards $\left(\mathrm{AUC}_{\mathrm{GIR}, 2-\mathrm{t}}\right)$ were estimated in the clamp setting to reflect the late-phase pharmacodynamic properties. In the pooled analysis of adults with T1D, $t_{\text {Late }} 50 \%$ GIRmax was 14.3 min shorter $(p<0.001)$ and $\mathrm{AUC}_{\mathrm{GIR}, 2-\mathrm{t}}$ was $10 \%$ smaller $(p<0.001)$ for faster aspart versus IAsp [16]. In subjects with T2D, the corresponding differences for offset and late glucose-lowering effect were 14.4 min earlier $(p=0.152)$ and $9 \%$ smaller $(p=0.083)$ for faster aspart versus IAsp [17]. Thus, although the mean treatment differences were very similar in subjects with T1D and T2D, statistical significance was not reached in the lower number of subjects with T2D [17]. Importantly, at the time of $t_{\text {Late } 50 \% \text { GIRmax }}$, considerable glucose-lowering effect is, by definition, still left and therefore $t_{\text {Late }} 50 \%$ GIRmax does not represent complete offset of action. However, as appears from Fig. 4, the decline in metabolic action is consistent for faster aspart and IAsp, therefore the 14 min earlier offset for faster aspart should be in the right range even for later time points. It is therefore concluded that faster aspart provides earlier offset of glucose-lowering effect compared with IAsp, which may potentially reduce the risk of late postprandial hypoglycaemia seen when glucose-lowering effect exceeds meal glucose absorption during the late postprandial phase.

\subsection{Overall Glucose-Lowering Effect}

In both subjects with T1D and T2D, total $\left(\mathrm{AUC}_{\mathrm{GIR}, 0-t}\right)$ and maximum $\left(\mathrm{GIR}_{\max }\right)$ glucose-lowering effect were comparable between faster aspart and IAsp, suggesting that both treatments provide the same total glucose-lowering effect when administered at similar doses [16, 17]. In subjects with T1D, the treatment ratios of faster aspart/IAsp were 0.98 (95\% CI 0.94-1.03, $p=0.426)$ and 1.01 (95\% CI $0.96-1.05, p=0.814)$, respectively [16]. In subjects with $\mathrm{T} 2 \mathrm{D}$, the treatment ratios of faster aspart/IAsp were 1.00 (95\% CI $0.92-1.08, p=0.960)$ and 1.03 (95\% CI 0.96-1.11, $p=0.373$ ), respectively [17].

The dose-response relationship for faster aspart was investigated in subjects with T1D within a dose range of $0.1-0.4 \mathrm{U} / \mathrm{kg}$ [19]. The increase in $\mathrm{AUC}_{\mathrm{GIR}, 0-\mathrm{t}}$ was proportional when doubling the faster aspart or IAsp dose from 0.1 to $0.2 \mathrm{U} / \mathrm{kg}$, while a less than proportional increase of $73 \%$ occurred when doubling the dose from 0.2 to $0.4 \mathrm{U} / \mathrm{kg}$ [19]. A previous dose-response trial with regular human insulin and insulin glulisine also showed a less than proportional increase in total glucose-lowering effect at doses up to $0.3 \mathrm{U} / \mathrm{kg}$ [55]. The relation between insulin concentration and glucose uptake is sigmoidal and the slope of the linear part, as well as the maximum level of glucose disposal, differ between individuals [56]. The finding of a less than proportional increase in glucose-lowering effect when doubling the dose to $0.3-0.4 \mathrm{U} /$ $\mathrm{kg}$ for several insulins, including faster aspart, can presumably be explained by saturation of insulin-stimulated glucose disposal in some subjects who reach their GIR $_{\max }$ at a dose level lower than the fixed maximum doses of 0.3-0.4 U/kg. Importantly, the lack of dose proportionality for glucose-lowering effect in a glucose clamp setting with fixed dose levels has limited consequences in clinical practice, where individual titration will ensure that patients stay on the linear part of their individual sigmoidal insulin dose-response curve [19].

\subsection{Pharmacodynamic Variability}

The variability in glucose-lowering effect between insulin doses is important for patients with diabetes aiming for optimal glycaemic control with minimal hypoglycaemia [57]. Withinsubject variability in glucose-lowering effect was investigated for faster aspart versus IAsp in a crossover trial where subjects received three single doses of $0.2 \mathrm{U} / \mathrm{kg}$ faster aspart or $0.2 \mathrm{U} / \mathrm{kg}$ IAsp on separate dosing days [19]. Within-subject variability for faster aspart, determined as the coefficient of variation, was 20-25\% for early glucose-lowering effect within 1 or $2 \mathrm{~h}$ after administration, $18 \%$ for $\mathrm{AUC}_{\mathrm{GIR}, 0-\mathrm{t}}$ and $19 \%$ for $\mathrm{GIR}_{\max }$ [19]. Within-subject variability did not differ statistically significantly between faster aspart and IAsp, and was in the same range for faster aspart as seen previously for regular human insulin, insulin lispro and insulin glulisine [48]. Due to the low within-subject variability in glucose-lowering effect, as previously observed with regular human insulin and previously developed rapid-acting insulins, patients may expect that the faster onset and greater initial glucose-lowering effect of faster aspart will be consistently seen from day to day.

\subsection{Mechanisms Behind the Increased Early Glucose-Lowering Effect with Faster Aspart}

As described in Sect. 8, the earlier onset and greater initial glucose-lowering effect of faster aspart versus IAsp leads to reduced PPG increment with faster aspart. The mechanisms behind were investigated in a meal test trial using triple-tracer methodology to determine PPG turnover in subjects with T1D [22]. During the first hour after meal ingestion, faster aspart induced not only a greater peripheral rate of glucose disappearance but also larger suppression of endogenous glucose production versus IAsp (Fig. 6). In healthy individuals, meal ingestion leads to rapid insulin secretion into the portal vein, aiming to lower BG concentrations via a reduction of hepatic glucose production and an increase in peripheral glucose uptake [3]. Appropriately balancing hepatic and peripheral effects is ensured through exposure of the liver to several-fold higher insulin levels than seen peripherally [58]. The fact that PPG reduction with faster aspart occurs partly via effects on the liver shows the importance of fast insulin 


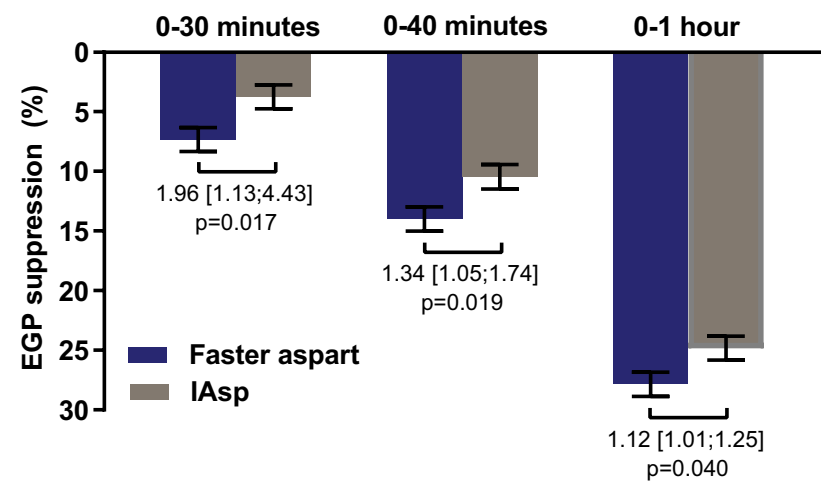

Fig. 6 Suppression of endogenous glucose production for faster aspart versus IAsp during a meal test after individualised subcutaneous dosing $(0.06-0.28 \mathrm{U} / \mathrm{kg})$ in subjects with T1D. Bars show LS mean \pm standard error. Treatment comparisons show treatment ratios of faster aspart/IAsp [95\% CI] and $p$ values. $C I$ confidence interval, EGP endogenous glucose production, IAsp insulin aspart, LS Mean least square mean. T1D type 1 diabetes. From Basu et al. [22]

absorption. Accordingly, it was recently shown that with faster aspart compared with IAsp, the PPG excursion following a mixed meal in subjects with T1D was considerably closer to the PPG excursion seen in healthy subjects investigated in the same study [59].

\section{Pharmacological Properties of Faster Aspart in Continuous Subcutaneous Insulin Infusion (CSII)}

As CSII use is increasing in diabetes, it is relevant to evaluate the performance of faster aspart administered by insulin pump [60]. The pharmacological characteristics of faster aspart in a CSII setting were investigated in subjects with T1D receiving a $0.15 \mathrm{U} / \mathrm{kg}$ bolus via CSII on top of a $0.02 \mathrm{U} / \mathrm{kg} / \mathrm{h}$ basal rate [20]. In line with results for subcutaneous injection, the pharmacokinetic (Fig. 7) and pharmacodynamic profiles of the bolus dose were both left-shifted for faster aspart versus IAsp. Onset of exposure $\left(t_{\text {Early } 50 \% C \max }\right)$ occurred 11.8 min earlier $(p<0.001), \mathrm{AUC}_{\text {IAsp }, 0-30 \min }$ was approximately threefold larger $(p<0.001)$ and $t_{\text {Late }} 50 \% C \max$ occurred 35.4 min earlier $(p<0.001)$ for faster aspart versus IAsp [20]. Likewise, $t_{\text {Early } 50 \% \text { GIRmax }}$ occurred $11.1 \mathrm{~min}$ earlier $(p<0.001), \mathrm{AUC}_{\mathrm{GIR}, 0-30 \mathrm{~min}}$ was approximately twofold greater $(p=0.002)$ and $t_{\text {Late } 50 \% \text { GIRmax }}$ occurred $24.0 \mathrm{~min}$ earlier $(p=0.002)$ for faster aspart versus IAsp [20]. $\mathrm{AUC}_{\mathrm{IAsp}, 0-\mathrm{t}}$ and $\mathrm{AUC}_{\mathrm{GIR}, 0-\mathrm{t}}$ from the bolus dose were both similar for faster aspart and IAsp. The treatment ratios of faster aspart/IAsp were 0.97 (95\% CI 0.90-1.05, $p=0.477$ ) and 1.04 (95\% CI $0.95-1.13, p=0.427)$, respectively [20]. In conclusion, faster aspart administered via CSII provides accelerated onset, greater initial exposure and glucose-lowering effect, and earlier offset relative to IAsp. Thus, in a CSII setting, the faster aspart pharmacological profile better

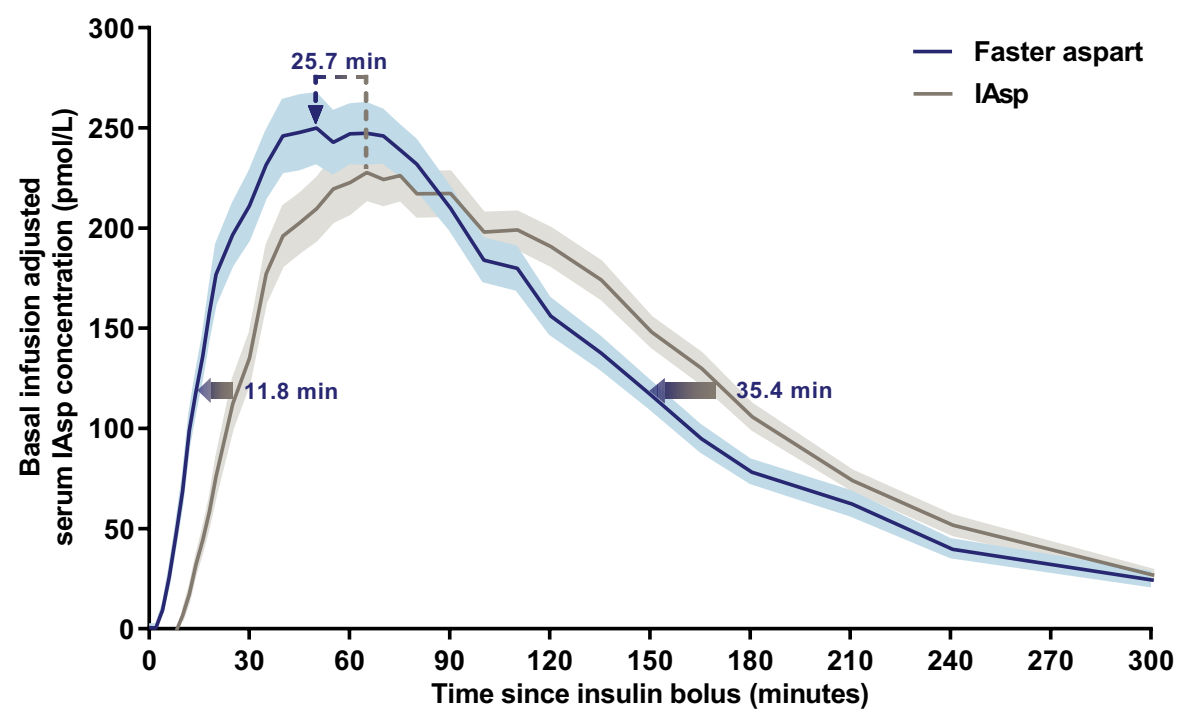

Fig. 7 Mean 5-h serum IAsp concentration-time profiles after a bolus dose of $0.15 \mathrm{U} / \mathrm{kg}$ faster aspart or IAsp administered by CSII on top of a basal infusion. Variability bands show the standard error of the mean. The blue/grey arrows indicate that the estimated onset and offset of exposure occurred earlier for faster aspart than for IAsp,

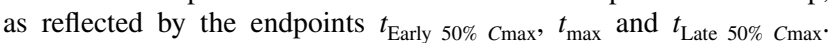

CSII continuous subcutaneous insulin infusion, IAsp insulin aspart, $t_{\text {Early } 50 \% \text { Cmax }}$ time to $50 \%$ of maximum concentration in the early part of the pharmacokinetic profile, $t_{\max }$ time to maximum concentration, $t_{\text {Late } 50 \% \text { Cmax }}$ time to $50 \%$ of maximum concentration in the late part of the pharmacokinetic profile, $U$ units. Modified from Heise et al. [20] 
approximates mealtime insulin secretion in healthy subjects, as observed after subcutaneous injection.

\section{Different Patient Populations, Injection Regions and Insulin Antibody Levels}

\subsection{Children and Adolescents}

Faster aspart was compared with IAsp in a pharmacokinetic and meal test trial in children (6-11 years), adolescents (12-17 years) and adults with T1D [23]. In children and adolescents, onset of appearance occurred approximately twice as fast, $t_{\text {Early } 50 \% ~ C \max }$ was $\sim 7$ min shorter and $\mathrm{AUC}_{\mathrm{IAsp}, 0-30 \mathrm{~min}}$ was 78-98\% greater for faster aspart versus IAsp (Fig. 8).
$\mathrm{AUC}_{\mathrm{IAsp}, 0-\mathrm{t}}$ for faster aspart was $41 \%$ lower in children $(p<0.001)$ and $22 \%$ lower in adolescents $(p=0.002)$ versus adults. In a linear mixed model with period, age group, treatment, and interaction between age group and treatment as fixed effects, the $p$ value for interaction between age group and treatment was 0.481 [23]. Thus, the age effect on $\mathrm{AUC}_{\mathrm{IAsp}, 0-\mathrm{t}}$ did not differ statistically significantly between faster aspart and IAsp. Furthermore, the age effect on $\mathrm{AUC}_{\mathrm{IAsp}, 0-\mathrm{t}}$ is not considered clinically important since individual titration of faster aspart must be carried out. Interestingly, all subjects received $0.2 \mathrm{U} / \mathrm{kg}$ body weight irrespective of age, and the age effect on $\mathrm{AUC}_{\text {IAsp,0-t }}$ largely followed the age group differences in absolute dose (mean of 8.3, 12.8 and $15.6 \mathrm{U}$ in children, adolescents and adults, respectively). It was therefore speculated that the increased total exposure
Fig. 8 Onset of exposure (a) and early exposure (b) for faster aspart versus IAsp after a subcutaneous dose of $0.2 \mathrm{U} / \mathrm{kg}$ in children and adolescents with T1D. ${ }^{a}$ Faster aspart-IAsp. ${ }^{b}$ For treatment comparison of faster aspart versus IAsp. ${ }^{\mathrm{c}}$ Faster aspart/IAsp. $A U C$ area under the curve, $C I$ confidence interval, IAsp insulin aspart, LS Mean least square mean, $T 1 D$ type 1 diabetes, $t_{\text {Early } 50 \% \text { Cmax }}$ time to $50 \%$ of maximum concentration in the early part of the pharmacokinetic profile, $t_{\max }$ time to maximum concentration, $U$ units. Data from Fath et al. [23]
(A) Endpoint

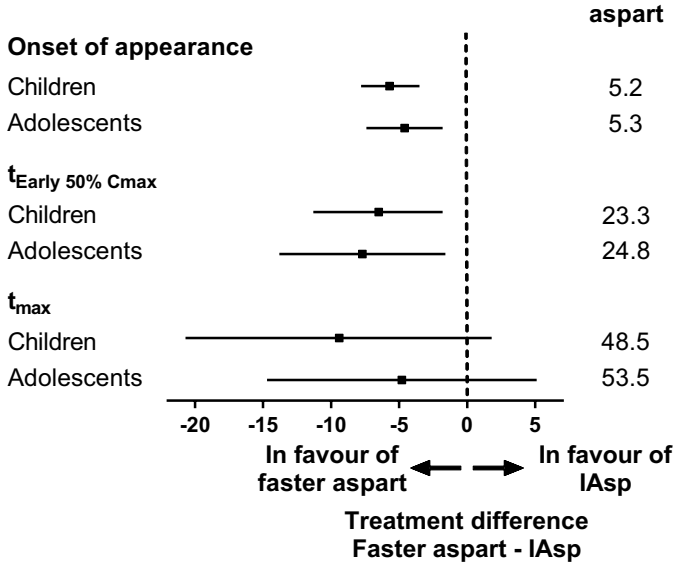

(B) Endpoint

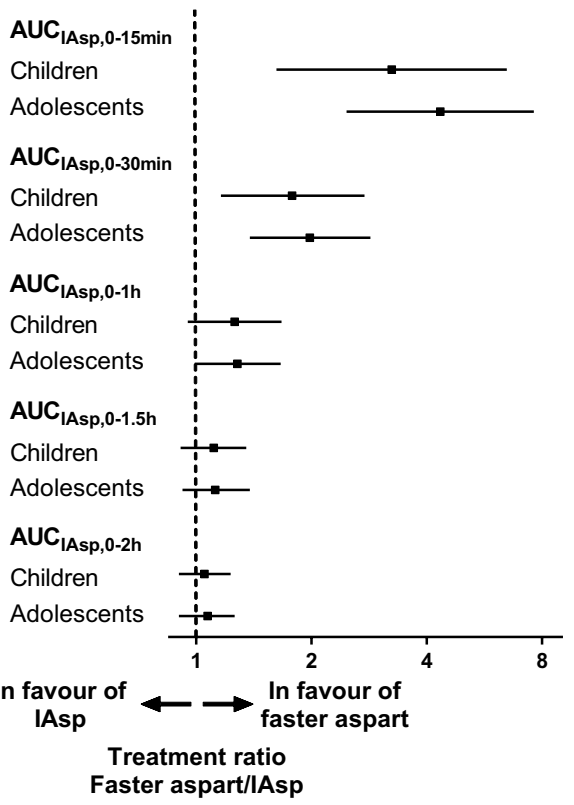

\begin{tabular}{|c|c|c|c|}
\hline LS Mean & ninutes) & Treatment & P-value \\
\hline $\begin{array}{l}\text { Faster } \\
\text { aspart }\end{array}$ & $\begin{array}{l}\text { Insulin } \\
\text { aspart }\end{array}$ & $\begin{array}{l}\text { difference }^{a} \\
{[95 \% \mathrm{Cl}]}\end{array}$ & \\
\hline 5.2 & 9.8 & $-4.6[-7.4 ;-1.8]$ & 0.004 \\
\hline 5.3 & 11.0 & $-5.7[-7.8 ;-3.5]$ & $<0.001$ \\
\hline 23.3 & 30.9 & $-7.7[-13.8 ;-1.6]$ & 0.019 \\
\hline 24.8 & 31.3 & $-6.5[-11.3 ;-1.8]$ & 0.012 \\
\hline 48.5 & 53.3 & $-4.8[-14.7 ; 5.1]$ & 0.300 \\
\hline 53.5 & 62.9 & $-9.4[-20.7 ; 1.8]$ & 0.093 \\
\hline $\begin{array}{l}\text { avour of } \\
\text { IAsp }\end{array}$ & & & \\
\hline
\end{tabular}
Faster Insulin $[95 \% \mathrm{Cl}]$ aspart aspart

$\begin{array}{llll}7.3 & 2.3 & 3.24[1.62 ; 6.47] & 0.003\end{array}$

$\begin{array}{llll}7.3 & 1.7 & 4.34[2.47 ; 7.62] & <0.001\end{array}$

$40.6 \quad 22.8 \quad 1.78[1.16 ; 2.75] \quad 0.013$

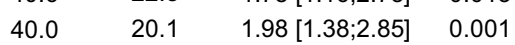

$\begin{array}{llll}140.6 & 111.5 & 1.26[0.95 ; 1.67] & 0.095\end{array}$

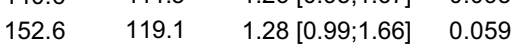

$\begin{array}{llll}225.7 & 203.3 & 1.11[0.91 ; 1.35] & 0.265\end{array}$

$\begin{array}{llll}261.8 & 233.3 & 1.12[0.92 ; 1.38] & 0.242\end{array}$

$\begin{array}{llll}284.5 & 270.5 & 1.05[0.90 ; 1.23] & 0.504\end{array}$

$346.0 \quad 324.1 \quad 1.07[0.90 ; 1.26] \quad 0.405$ 
with increasing age might reflect that the volume of distribution does not develop closely according to increases in body weight from childhood and adolescence to adulthood [23]. In response to a standardised liquid meal, the average glucose excursion during the first 1 and $2 \mathrm{~h}$ and the maximum glucose excursion were all reduced in children by $1.2-1.6 \mathrm{mmol} / \mathrm{L}$ for faster aspart versus IAsp $(p=0.005$, $p=0.028$ and $p=0.044$, respectively). A trend in the same direction was seen in adolescents $(0.2-0.6 \mathrm{mmol} / \mathrm{L})$, which was not statistically significant [23].

\subsection{Elderly}

In a glucose clamp trial comparing faster aspart with IAsp in elderly subjects ( $\geq 65$ years) and younger adults (18-35 years) with T1D, onset of appearance occurred approximately twice as fast ( 3 min earlier; $p<0.001$ ), $t_{\text {Early } 50 \% C \max }$ was 10 min shorter $(p<0.001)$ and initial exposure up to $2 \mathrm{~h}$ after dosing was greater for faster aspart versus IAsp in the elderly subjects [24]. Similarly, onset of action occurred 10 min earlier, $t_{\text {Early } 50 \% \text { GIRmax }}$ was 6 min shorter and initial glucose-lowering effect up to $2 \mathrm{~h}$ after dosing was greater for faster aspart versus IAsp (Fig. 9). The effect of faster aspart versus IAsp on the pharmacokinetic/pharmacodynamic endpoints did not differ statistically significantly between elderly and younger adults [24]. Thus, faster aspart should also be able to reduce PPG excursion compared with previously developed rapid-acting insulins in elderly patients with diabetes.

\subsection{Individuals with Renal or Hepatic Impairment}

Renal and hepatic impairment may potentially affect drug clearance and/or metabolism $[61,62]$. The pharmacokinetic properties of faster aspart have not been investigated in subjects with renal or hepatic impairment. The only change in faster aspart versus IAsp relates to the subcutaneous absorption process, and the IAsp molecule in faster aspart has not been modified compared with the original IAsp formulation [13]. Furthermore, receptor-mediated clearance is the main pathway for clearance of IAsp molecules. Therefore, pharmacokinetic results on IAsp in renal and hepatic impairment can also be used as representative for faster aspart. It was shown that there is no impact of renal impairment (as assessed by creatinine clearance) or hepatic impairment (as assessed by Child-Pugh score) on the pharmacokinetics of IAsp [63]. Consequently, it is concluded that the pharmacokinetic characteristics of faster aspart are also not affected, to any clinically significant extent, by renal or hepatic impairment.

\subsection{Japanese Individuals}

Since race and ethnicity may affect the pharmacological characteristics of insulin products, the pharmacological properties of faster aspart were compared with IAsp in a glucose clamp trial in Japanese subjects with T1D [25, 64]. The pharmacokinetic/pharmacodynamic profiles were leftshifted for faster aspart versus IAsp (Fig. 10). For faster aspart relative to IAsp, onset of appearance occurred $4.1 \mathrm{~min}$
Fig. 9 Onset of glucoselowering effect (a) and early glucose-lowering effect (b) for faster aspart versus IAsp after a subcutaneous dose of $0.2 \mathrm{U} / \mathrm{kg}$ in elderly subjects with T1D. ${ }^{a}$ Faster aspart-IAsp. ${ }^{b}$ For treatment comparison of faster aspart versus IAsp. ${ }^{\mathrm{c}}$ Faster aspart/IAsp. AUC area under the curve, $C I$ confidence interval, GIR glucose infusion rate, IAsp insulin aspart, LS Mean least square mean, $T 1 D$ type 1 diabetes, $t_{\text {Early } 50 \% \text { GIRmax }}$ time to $50 \%$ of maximum glucose infusion rate in the early part of the glucose infusion rate profile, $t_{\text {GIRmax }}$ time to maximum glucose infusion rate, $U$ units. Data from Heise et al. [24]

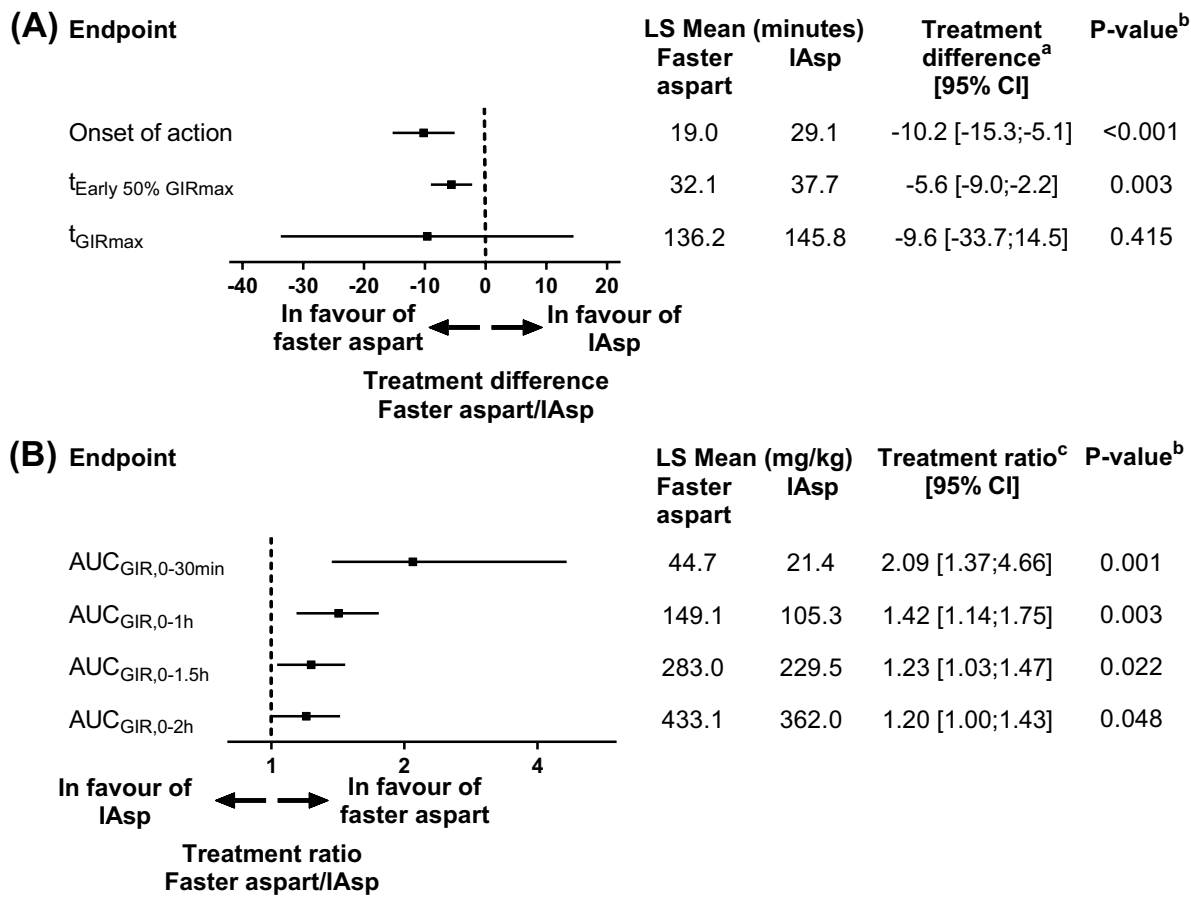




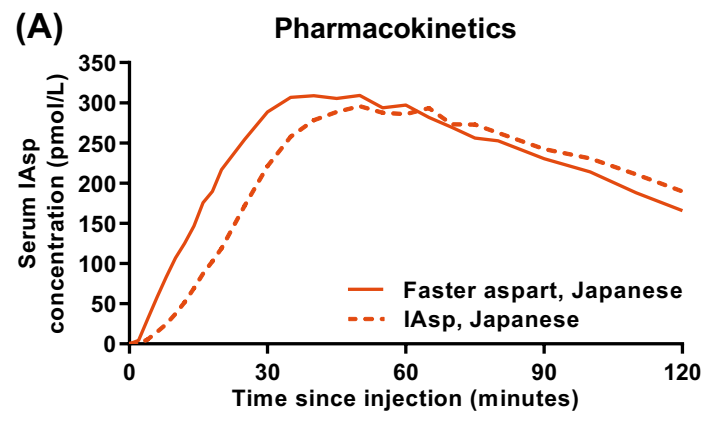

Fig. 10 Mean 2-h serum IAsp concentration-time profiles (a) and mean 2-h glucose-lowering effect profiles (b) for faster aspart versus IAsp after a subcutaneous dose of $0.2 \mathrm{U} / \mathrm{kg}$ in Japanese subjects with

earlier $(p<0.001), t_{\text {Early } 50 \% C \max }$ was 10.2 min shorter $(p<0.001)$ and $\mathrm{AUC}_{\mathrm{IAsp}, 0-30 \mathrm{~min}}$ was $94 \%$ greater $(p<0.001)$ [25]. Likewise, onset of action occurred 5.3 min earlier $(p=0.001), t_{\text {Early } 50 \% \text { GIRmax }}$ was 10.0 min shorter $(p<0.001)$ and $\mathrm{AUC}_{\mathrm{GIR}, 0-30 \min }$ was $110 \%$ greater $(p=0.002)$ [25]. The corresponding differences for faster aspart relative to IAsp in Caucasians were $4.9 \mathrm{~min}$ earlier onset of appearance, $9.5 \mathrm{~min}$ shorter $t_{\text {Early } 50 \% C \max }, 101 \%$ greater $\mathrm{AUC}_{\mathrm{IAsp}, 0-30 \mathrm{~min}}, 4.9 \mathrm{~min}$ earlier onset of action, 9.5 min shorter $t_{\text {Early } 50 \% \text { GIRmax }}$ and $74 \%$ greater $\mathrm{AUC}_{\mathrm{GIR}, 0-30 \mathrm{~min}}$ (all $p<0.001$ ). Thus, in accordance with results shown in Caucasian subjects, faster aspart in Japanese subjects also better resembles healthy postprandial insulin secretion compared with previously developed rapid-acting insulins.

\subsection{Different Injection Regions}

Insulin can be administered subcutaneously in various injection regions, however potentially with different pharmacokinetic profiles [65-67]. Therefore, a trial in healthy males investigated the pharmacokinetics of faster aspart administered subcutaneously in the abdomen, upper arm or thigh (Fig. 11) [26]. Onset of appearance was 3 min, $t_{\text {Early } 50 \% ~ C \max }$ was $\sim 20 \mathrm{~min}$ and $t_{\max }$ was $\sim 55 \mathrm{~min}$ for faster aspart independent of injection region. Early exposure within the first 1 or $2 \mathrm{~h}$ after administration and $C_{\max }$ were $20-30 \%$ lower for the thigh versus the abdomen and upper arm. $\mathrm{AUC}_{\mathrm{IAsp}, 0-\mathrm{t}}$ was comparable for all three injection regions [26]. Based on these results, the ultra-fast pharmacokinetic properties of faster aspart are most pronounced when administered in the abdomen or upper arm compared with the thigh.

\subsection{Effect of Anti-Insulin Antibodies}

The impact of anti-IAsp antibodies on the pharmacokinetics/pharmacodynamics of faster aspart and IAsp was investigated based on four clinical pharmacology trials supplemented with results from a phase III trial [21]. For both

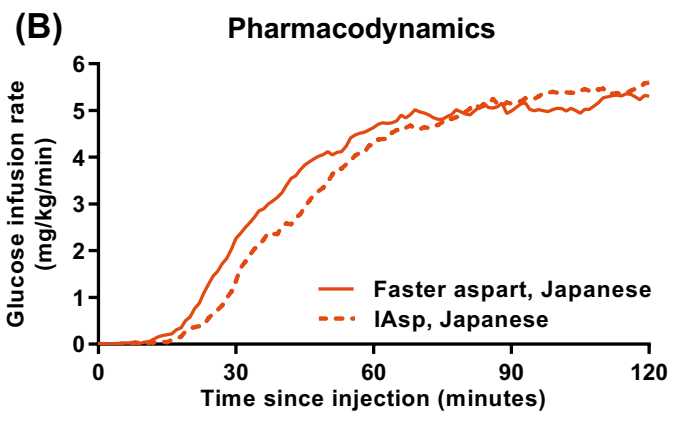

T1D. IAsp insulin aspart, T1D type 1 diabetes, $U$ units. From Shiramoto et al. [25]

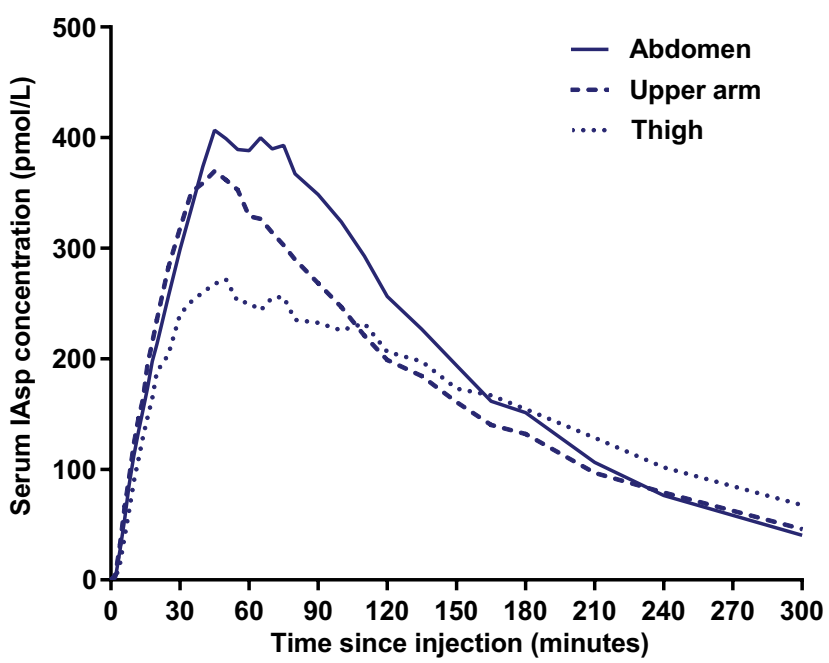

Fig. 11 Mean 5-h serum IAsp concentration-time profiles for $0.2 \mathrm{U} / \mathrm{kg}$ faster aspart administered subcutaneously in the abdomen, upper arm or thigh in healthy male subjects. IAsp insulin aspart, $U$ units. From Hövelmann et al. [26]

faster aspart and IAsp, overall exposure was approximately twofold greater for total versus free IAsp, which could at least partly be explained by the presence of anti-IAsp antibodies. Thus, higher anti-IAsp antibody levels were associated with lower ratio of free/total IAsp for $\mathrm{AUC}_{\mathrm{IAsp}, 0-\mathrm{t}}$ [21]. It was also shown that initial glucose-lowering effect within $1 \mathrm{~h}$ post-dose was consistently greater for faster aspart versus IAsp irrespective of anti-IAsp antibody levels, and there was essentially no correlation between anti-IAsp antibody level and PPG increment at $1 \mathrm{~h}$ in a meal test [21]. Based on these findings, it is concluded that faster aspart provides accelerated pharmacological properties versus IAsp independent of the anti-IAsp antibody level. This is in line with several other trials showing no clinically significant impact of anti-insulin antibodies on insulin pharmacodynamics, efficacy or safety [21, 68-70]. 


\section{Clinical Consequences of Faster Aspart Pharmacological Properties}

\subsection{Multiple Daily Injection Therapy}

The improved pharmacological properties of faster aspart have been shown in several phase III trials to result in better PPG control and at least as efficient overall glycaemic control with similar or reduced overall risk of hypoglycaemia compared with IAsp when used as multiple daily injection therapy and administered at meal initiation (Table 1).

In subjects with $\mathrm{T} 1 \mathrm{D}$, the efficacy and safety of faster aspart were compared with IAsp in a basal-bolus regimen in a 52-week trial with insulin detemir and in a 26-week trial with insulin degludec $[28,30,31]$. PPG increment in a meal test was consistently reduced by faster aspart versus IAsp at $1 \mathrm{~h}$ post-meal in both trials, and also at 30 min post-meal in the 26 -week trial and at $2 \mathrm{~h}$ postmeal after 26 weeks of the 52-week trial (Fig. 12a) [28, $30,31]$. In the 52-week trial, $\mathrm{HbA}_{1 \mathrm{c}}$ was reduced, for faster aspart versus IAsp, by $0.15 \%$ after 26 weeks and $0.10 \%$ after 52 weeks, while non-inferiority in $\mathrm{HbA}_{1 \mathrm{c}}$ was demonstrated for faster aspart versus IAsp in the 26-week trial (Table 1). The rate of overall severe or BG-confirmed hypoglycaemia was comparable for faster aspart and IAsp in both trials (Table 1).

In subjects with T2D, a 26-week trial and a 16-week trial both compared faster aspart with IAsp in a basal-bolus regimen and showed that PPG increment in a meal test was reduced by faster aspart versus IAsp at $1 \mathrm{~h}$ post-meal with non-inferiority in $\mathrm{HbA}_{1 \mathrm{c}}$, i.e. essentially similar results as shown in T1D (Table 1) [32,33]. While the rate of overall

Table 1 Summary of efficacy and hypoglycaemia results in phase III trials with faster aspart

\begin{tabular}{|c|c|c|c|c|c|c|c|c|}
\hline \multirow[t]{2}{*}{ Study name } & \multirow[t]{2}{*}{$\begin{array}{l}\text { Study popula- } \\
\text { tion }\end{array}$} & \multirow[t]{2}{*}{$\begin{array}{l}\text { Treatment dura- } \\
\text { tion; treatment } \\
\text { regimen }\end{array}$} & \multirow[t]{2}{*}{ Comparator } & \multicolumn{3}{|c|}{$\begin{array}{l}\text { Difference in efficacy with faster aspart vs. } \\
\text { comparator (estimated treatment difference) }\end{array}$} & \multicolumn{2}{|c|}{$\begin{array}{l}\text { Difference in rate of } \\
\text { hypoglycaemia with faster } \\
\text { aspart vs. comparator ( } \% \\
\text { difference) }\end{array}$} \\
\hline & & & & $\mathrm{HbA}_{1 \mathrm{c}}(\%)$ & $\begin{array}{l}\Delta \mathrm{PPG}_{1 \mathrm{~h}} \\
(\mathrm{mmol} / \mathrm{L})\end{array}$ & $\begin{array}{l}\Delta \mathrm{PPG}_{2 \mathrm{~h}} \\
(\mathrm{mmol} / \mathrm{L})\end{array}$ & $\begin{array}{l}\text { Severe } \\
\text { hypogly- } \\
\text { caemia }\end{array}$ & $\begin{array}{l}\text { Severe or } \\
\text { BG-confirmed } \\
\text { hypoglycaemia }\end{array}$ \\
\hline \multicolumn{9}{|l|}{ MDI therapy } \\
\hline Onset 1 [30] & T1D & $\begin{array}{l}26 \text { weeks; BB } \\
\text { IDet }\end{array}$ & IAsp & $\begin{array}{l}\mathbf{- 0 . 1 5} \text {; non- } \\
\text { inferior; } \\
\text { significant }\end{array}$ & -1.18 & -0.67 & ND & $1 \uparrow$ \\
\hline Onset 1 [28] & T1D & $\begin{array}{l}52 \text { weeks; BB } \\
\text { IDet }\end{array}$ & IAsp & $\begin{array}{l}\text {-0.10; sig- } \\
\text { nificant }\end{array}$ & -0.91 & -0.42 & $21 \downarrow$ & $1 \uparrow$ \\
\hline Onset 8 [31] & T1D & $\begin{array}{l}26 \text { weeks; BB } \\
\text { IDeg }\end{array}$ & IAsp & $\begin{array}{l}-0.02 ; \text { non- } \\
\text { inferior }\end{array}$ & -0.90 & -0.35 & ND & NS \\
\hline Onset 2 [32] & $\begin{array}{l}\text { T2D; treated } \\
\text { with basal } \\
\text { insu- } \\
\text { lin + OAD(s) }\end{array}$ & $\begin{array}{l}26 \text { weeks; BB } \\
\text { IGlar + OAD(s) }\end{array}$ & IAsp & $\begin{array}{l}-0.02 \text {; non- } \\
\text { inferior }\end{array}$ & -0.59 & -0.36 & $25 \uparrow$ & $9 \uparrow$ \\
\hline Onset 9 [33] & $\begin{array}{l}\text { T2D; treated } \\
\text { with } \mathrm{BB}\end{array}$ & $\begin{array}{l}16 \text { weeks; BB } \\
\text { IDeg } \pm \text { met- } \\
\text { formin }\end{array}$ & IAsp & $\begin{array}{l}-0.04 \text {; non- } \\
\text { inferior }\end{array}$ & -0.40 & ND & ND & $19 \downarrow$ \\
\hline Onset 3 [34] & $\begin{array}{l}\text { T2D; treated } \\
\text { with basal } \\
\text { insu- } \\
\text { lin + OAD(s) }\end{array}$ & $\begin{array}{l}18 \text { weeks; } \\
\text { BB }+ \text { metformin }\end{array}$ & $\begin{array}{l}\text { Basal } \\
\text { only }+ \text { met- } \\
\text { formin }\end{array}$ & $\begin{array}{l}-\mathbf{0 . 9 4} \text {; supe- } \\
\text { rior }\end{array}$ & ND & ND & $789 \uparrow$ & $724 \uparrow$ \\
\hline \multicolumn{9}{|l|}{ CSII therapy } \\
\hline Onset 5 [29] & $\begin{array}{l}\text { T1D; treated } \\
\text { via CSII }\end{array}$ & 26 weeks & IAsp & $\begin{array}{l}\mathbf{0 . 0 9} \text {; non- } \\
\text { inferior; } \\
\text { significant }\end{array}$ & -0.91 & -0.90 & ND & $0 \leftrightarrow$ \\
\hline
\end{tabular}

Results shown in bold indicate a statistically significant difference between faster aspart and comparator. Arrows for hypoglycaemia indicate the numerical direction of the treatment difference

$B B$ basal-bolus, $B G$ blood glucose, $C S I I$ continuous subcutaneous insulin infusion, $\triangle P P G_{l h}$ 1-h postprandial glucose increment, $\triangle P P G_{2 h}$ 2-h postprandial glucose increment, $H b A_{l c}$ glycosylated haemoglobin, IAsp insulin aspart, IDeg insulin degludec, IDet insulin detemir, IGlar insulin glargine, $M D I$ multiple daily injection, $N D$ not determined, $N S$ not significant (but no estimate provided), $O A D$ oral antidiabetic drug, $T 1 D$ type 1 diabetes, $T 2 D$ type 2 diabetes 

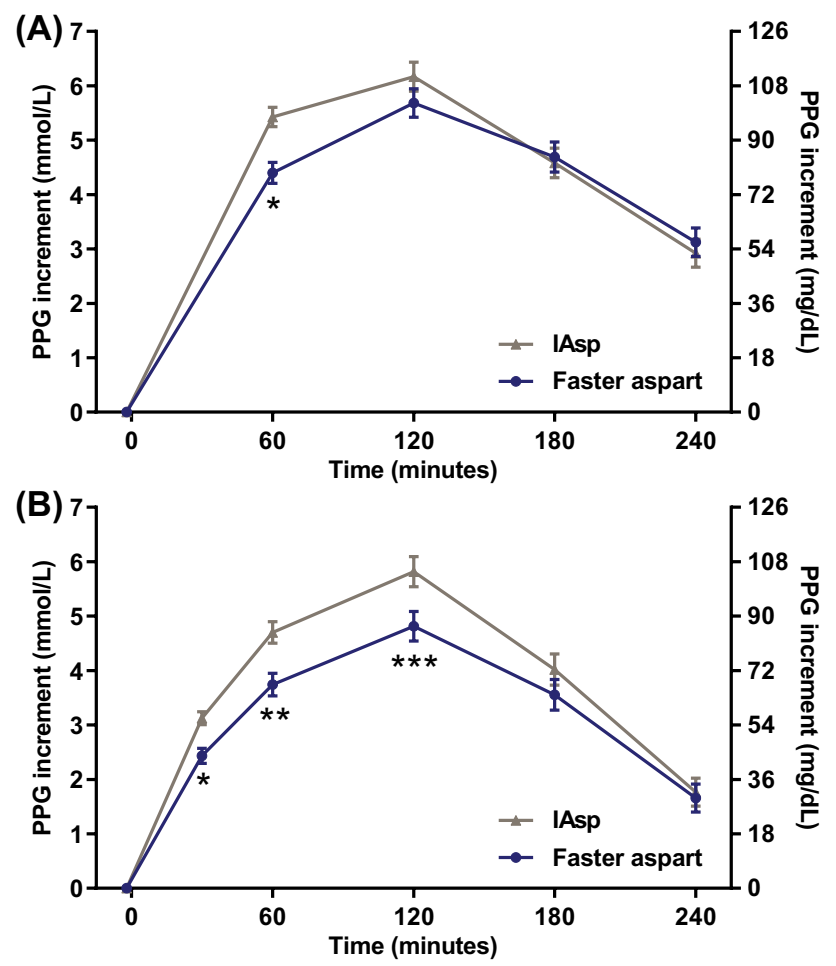

Fig. 12 PPG increment for faster aspart versus IAsp in subjects with T1D during a 4-h meal test conducted after 52 weeks of multiple daily injection therapy (a) or after 16 weeks of CSII treatment (b). Subjects received a bolus dose of $0.1 \mathrm{U} / \mathrm{kg}$ followed by a standardised liquid meal (approx. $80 \mathrm{~g}$ carbohydrate) consumed within $12 \mathrm{~min}$. Error bars show \pm standard error of the mean. $* p<0.001, * * p=0.001$, $* * * p=0.01$. CSII continuous subcutaneous insulin infusion, IAsp insulin aspart, $P P G$ postprandial glucose, T1D type 1 diabetes, $U$ units. From Mathieu et al. [28] and Klonoff et al. [29]

severe or BG-confirmed hypoglycaemia was comparable for faster aspart and IAsp in the 26-week trial, a significantly lower hypoglycaemia rate was observed for faster aspart in the 16-week trial [32,33]. An 18-week trial investigated the addition of faster aspart to basal insulin and metformin in subjects with T2D [34]. Expectedly, superiority in $\mathrm{HbA}_{1 \mathrm{c}}$ reduction (Table 1) and improvement of $2.48 \mathrm{mmol} / \mathrm{L}$ in average self-measured 2-h PPG for all meals were shown for faster aspart versus basal-only therapy, with a several-fold higher rate of severe or BG-confirmed hypoglycaemia when adding faster aspart to basal insulin (Table 1).

The efficacy and safety of faster aspart were also evaluated in children and adolescents (1-17 years) with T1D in a 26-week trial with faster aspart or IAsp in a basal-bolus regimen with insulin degludec [35]. PPG increment at $1 \mathrm{~h}$, based on self-measured BG, was reduced for faster aspart versus IAsp at breakfast, dinner and across all meals, and $\mathrm{HbA}_{1 \mathrm{c}}$ was reduced by $0.17 \%$ for faster aspart versus IAsp, with a comparable overall rate of severe or BG-confirmed hypoglycaemia [35]. These results suggest that also in children and adolescents, the accelerated absorption of faster aspart leads to improved PPG control, and at least as efficient overall glycaemic control with similar overall risk of hypoglycaemia compared with IAsp.

The ultra-fast pharmacological characteristics of faster aspart may also allow for post-meal dosing. Faster aspart administered 20 min after meal initiation was investigated in several phase III trials including children, adolescents and adults with T1D [30, 31, 35]. PPG increment in a meal test was higher with post-meal faster aspart versus mealtime IAsp up to $1 \mathrm{~h}$ post-meal. Nonetheless, post-meal faster aspart provided a non-inferior $\mathrm{HbA}_{1 \mathrm{c}}$ reduction and comparable overall rate of severe or BG-confirmed hypoglycaemia versus mealtime IAsp [30, 31, 35]. These findings suggest that post-meal administration of faster aspart could be an alternative in children, who often have irregular eating patterns, and in other situations where actual food intake is difficult to estimate in advance.

\subsection{CSII}

With accelerated insulin pharmacokinetics, as seen for faster aspart, it may be possible to further utilise the features of insulin pumps [71]. Pump compatibility of faster aspart versus IAsp over 6 weeks was investigated in 37 subjects with T1D. There were no observations of microscopically confirmed occlusions of the infusion set, suggesting comparable compatibility of faster aspart and IAsp in CSII [42]. An exploratory trial in 43 subjects with T1D showed that in a meal test following 2 weeks of CSII with faster aspart or IAsp, the average BG increment across the first $2 \mathrm{~h}$ after meal ingestion was $0.99 \mathrm{mmol} / \mathrm{L}$ lower with faster aspart versus IAsp [43]. Finally, in a 16-week phase III trial in adults with T1D investigating the efficacy and safety of faster aspart in CSII, the PPG increment in a meal test at the end of treatment was reduced by faster aspart versus IAsp at $30 \mathrm{~min}, 1$ and $2 \mathrm{~h}$ post-meal (Fig. 12b) [29]. $\mathrm{HbA}_{1 \mathrm{c}}$ was noninferior for faster aspart versus IAsp, although the 0.09\% difference in favour of IAsp was statistically significant, and the rate of overall severe or BG-confirmed hypoglycaemia was similar for faster aspart and IAsp (Table 1) [29]. Thus, it appears that the improved PPG control with faster aspart versus previously developed rapid-acting insulin seen after subcutaneous injection also pertains to treatment via CSII. As more advanced insulin pump systems become available, it is possible that the accelerated absorption of faster aspart will be even more important for future insulin application systems, including closed-loop and artificial pancreas systems $[71,72]$. Still, given the relatively limited clinical experience with the use of faster aspart in pumps, larger-scale clinical trials and/or accumulated real-world experience are needed to fully unravel both the safety profile and the clinical potential of faster aspart in pump use [73]. 


\section{Potential Risk Factors and Limitations Associated with Faster Aspart}

In all clinical trials, faster aspart was safe and well tolerated, which was expected since the IAsp molecule is unchanged in the faster aspart formulation and the two added excipients in faster aspart versus the original IAsp formulation are also assessed to be safe (see Sect. 2).

With an ultra-fast-acting insulin, there is a risk that the glucose-lowering effect profile may become too fast to match the glucose absorption from the meal, particularly in case of large and/or high-fat meals $[5,11]$. In some trials, faster aspart has been associated with a slightly increased risk of hypoglycaemia during the first $1-2 \mathrm{~h}$ after meal initiation, although this time period is characterised by a generally low frequency of hypoglycaemia [28-30, 32]. Another potential concern could be the risk of late under-insulinisation and resulting hyperglycaemia. However, this seems not to be an issue with faster aspart, at least up to $4 \mathrm{~h}$ post-meal (Fig. 12). Rather, a reduced risk of hypoglycaemia from 3 to $4 \mathrm{~h}$ post-meal was shown with faster aspart versus IAsp, indicating that the faster aspart action profile better matches the need for glucose disposal during the late postprandial period [31]. Nevertheless, a given duration of insulin action cannot fit all types and sizes of meals. In the majority of trials investigating the PPG excursion with faster aspart versus IAsp in a meal test, subjects ingested a standard liquid meal $[23,28-33]$. It is also important to establish the best dosing schedules of faster aspart for mixed, high-fat or highcarbohydrate meals with respect to injection-meal interval and the potential need for additional doses. Interestingly, several trials have investigated PPG control with mealtime faster aspart versus mealtime IAsp following subjects' own individual meals. In subjects with T2D, change from baseline in self-measured 1-h PPG increment was statistically significantly in favour of faster aspart versus IAsp at lunch, main evening meal and across all meals [33]. In children and adolescents with T1D, changes from baseline in 1- and 2-h interstitial glucose increments assessed by continuous glucose monitoring were statistically significantly in favour of faster aspart versus IAsp at breakfast, main evening meal and across all meals [35]. Furthermore, in adults with T1D using CSII, changes from baseline in mean interstitial glucose increment over 1 or $2 \mathrm{~h}$ were statistically significantly in favour of faster aspart versus IAsp at all individual meals and across meals [29]. As meal composition and timing may vary considerably between individuals and between different regions and countries, studies to investigate dosing schedules for faster aspart in relation to meal types will not be able to cover all meal regimens. Therefore, it is always important to take an individual approach to ensure the best match between insulin dosing regimen and meal habits.

The earlier onset and shorter duration of action of faster aspart should also be considered when using faster aspart via CSII. In a double-blind trial investigating faster aspart versus IAsp administered via CSII, continuous glucose monitoring showed higher nocturnal and pre-meal interstitial glucose concentrations with faster aspart than with IAsp [29]. It was hypothesized that the pump settings for both the basal rate and the bolus characteristics may have favoured IAsp and that adjustments might have been needed to adapt to the specific pharmacokinetics of faster aspart [29]. Thus, with faster aspart used in CSII, it must be considered in clinical practice if a different distribution between basal rate and bolus doses is needed and whether adjustments to the various bolus types are required compared with current practice for rapid-acting insulins [73].

\section{Conclusions}

Faster aspart is an ultra-fast-acting formulation of IAsp developed to provide accelerated absorption after subcutaneous administration. Faster aspart has consistently provided overall left-shifts of the pharmacokinetic/pharmacodynamic profiles compared with IAsp across different populations (adults with T1D or T2D, children and adolescents, the elderly and Japanese individuals) as well as in CSII. The improved pharmacological characteristics of faster aspart translate into superior PPG control and at least as efficient overall glycaemic control with a similar risk of hypoglycaemia compared with IAsp. Altogether, faster aspart may partly address the unmet need in patients with diabetes for mealtime insulins, with a pharmacokinetic profile that approaches healthy endogenous prandial insulin secretion.

Acknowledgements The authors would like to thank Carsten Roepstorff, PhD, CR Pharma Consult, Copenhagen, Denmark, for providing medical writing support funded by Novo Nordisk, and Naveen Rathor, MD, Novo Nordisk, for providing a medical accuracy review of the final draft.

\section{Compliance with Ethical Standards}

Conflict of interest This article was funded by Novo Nordisk. Hanne Haahr is an employee and shareholder of Novo Nordisk. Tim Heise is shareholder of Profil, which has received research funds from Adocia, Boehringer Ingelheim, Dance Pharmaceuticals, Eli Lilly, Gan \& Lee Pharmaceuticals, Johnson \& Johnson, Mars, MedImmune, Mylan, Nordic Bioscience, Novo Nordisk, Pfizer, Poxel, Saniona, Sanofi, Wockhardt and Zealand Pharma. In addition, Tim Heise is member of advisory panels for Mylan and Novo Nordisk and received speaker honoraria and travel grants from Eli Lilly and Novo Nordisk. 
Open Access This article is distributed under the terms of the Creative Commons Attribution-NonCommercial 4.0 International License (http://creativecommons.org/licenses/by-nc/4.0/), which permits any noncommercial use, distribution, and reproduction in any medium, provided you give appropriate credit to the original author(s) and the source, provide a link to the Creative Commons license, and indicate if changes were made.

\section{References}

1. Monnier L, Lapinski H, Colette C. Contributions of fasting and postprandial plasma glucose increments to the overall diurnal hyperglycemia of type 2 diabetic patients. Variations with increasing levels of $\mathrm{HbA}_{1 \mathrm{c}}$. Diabetes Care. 2003;26:881-5.

2. Woerle HJ, Neumann C, Zschau S, et al. Impact of fasting and postprandial glycemia on overall glycemic control in type 2 diabetes Importance of postprandial glycemia to achieve target HbA1c levels. Diabetes Res Clin Pract. 2007;77:280-5.

3. Saad A, Dalla Man C, Nandy DK, et al. Diurnal pattern to insulin secretion and insulin action in healthy individuals. Diabetes. 2012;61:2691-700.

4. Heise T. Getting closer to physiologic insulin secretion. Clin Ther. 2007;29:S161-5.

5. Heinemann L, Muchmore DB. Ultrafast-acting insulins: state of the art. J Diabetes Sci Technol. 2012;6:728-42.

6. Home PD. Plasma insulin profiles after subcutaneous injection: how close can we get to physiology in people with diabetes? Diabetes Obes Metab. 2015;17:1011-20.

7. American Diabetes Association. 9. Pharmacologic approaches to glycemic treatment: standards of medical care in diabetes-2019. Diabetes Care. 2019;42(Suppl 1):S90-102.

8. Sheldon B, Russell-Jones D, Wright J. Insulin analogues: an example of applied medical science. Diabetes Obes Metab. 2009; 11:5-19.

9. Home PD. The pharmacokinetics and pharmacodynamics of rapid-acting insulin analogues and their clinical consequences. Diabetes Obes Metab. 2012;14:780-8.

10. Luijf YM, van Bon AC, Hoekstra JB, Devries JH. Premeal injection of rapid-acting insulin reduces postprandial glycemic excursions in type 1 diabetes. Diabetes Care. 2010;33:2152-5.

11. Slattery D, Amiel SA, Choudhary P. Optimal prandial timing of bolus insulin in diabetes management: a review. Diabet Med. 2018;35:306-16.

12. Overmann H, Heinemann L. Injection-meal interval: recommendations of diabetologists and how patients handle it. Diabetes Res Clin Pract. 1999;43:137-42.

13. Kildegaard J, Buckley ST, Nielsen RH, et al. Elucidating the mechanism of absorption of fast-acting insulin aspart: the role of niacinamide. Pharm Res. 2019;36:49.

14. Biester T, Kordonouri O, Danne T. Pharmacological properties of faster-acting insulin aspart. Curr Diabetes Rep. 2017;17:101.

15. Senior P, Hramiak I. Fast-acting insulin aspart and the need for new mealtime insulin analogues in adults with type 1 and type 2 diabetes: a Canadian perspective. Can J Diabetes. 2019;43:515-23.

16. Heise T, Pieber TR, Danne T, Erichsen L, Haahr H. A pooled analysis of clinical pharmacology trials investigating the pharmacokinetic and pharmacodynamic characteristics of fast-acting insulin aspart in adults with type 1 diabetes. Clin Pharmacokinet. 2017;56:551-9.

17. Pieber TR, Svehlikova E, Brunner M, Halberg IB, Thomsen KMD, Haahr H. Fast-acting insulin aspart in subjects with type 2 diabetes: earlier onset and greater initial exposure and glucose-lowering effect compared with insulin aspart. Diabetes Obes Metab. 2019;21:2068-75.

18. Heise T, Hövelmann U, Brøndsted L, Adrian CL, Nosek L, Haahr H. Faster-acting insulin aspart: earlier onset of appearance and greater early pharmacokinetic and pharmacodynamic effects than insulin aspart. Diabetes Obes Metab. 2015;17:682-8.

19. Heise T, Stender-Petersen K, Hövelmann U, et al. Pharmacokinetic and pharmacodynamic properties of faster-acting insulin aspart versus insulin aspart across a clinically relevant dose range in subjects with type 1 diabetes mellitus. Clin Pharmacokinet. 2017;56:649-60.

20. Heise T, Zijlstra E, Nosek L, Rikte T, Haahr H. Pharmacological properties of faster-acting insulin aspart vs insulin aspart in patients with type 1 diabetes receiving continuous subcutaneous insulin infusion: a randomized, double-blind, crossover trial. Diabetes Obes Metab. 2017;19:208-15.

21. Haahr H, Pieber TR, Mathieu C, et al. Clinical pharmacology of fast-acting insulin aspart versus insulin aspart measured as free or total insulin aspart and the relation to anti-insulin aspart antibody levels in subjects with type 1 diabetes mellitus. Clin Pharmacokinet. 2019;58:639-49.

22. Basu A, Pieber TR, Hansen AK, et al. Greater early postprandial suppression of endogenous glucose production and higher initial glucose disappearance is achieved with fast-acting insulin aspart compared with insulin aspart. Diabetes Obes Metab. 2018;20:1615-22.

23. Fath M, Danne T, Biester T, Erichsen L, Kordonouri O, Haahr H. Faster-acting insulin aspart provides faster onset and greater early exposure vs insulin aspart in children and adolescents with type 1 diabetes mellitus. Pediatr Diabetes. 2017;18:903-10.

24. Heise T, Hövelmann U, Zijlstra E, Stender-Petersen K, Jacobsen $\mathrm{JB}, \mathrm{Haahr} \mathrm{H}$. A comparison of pharmacokinetic and pharmacodynamic properties between faster-acting insulin aspart and insulin aspart in elderly subjects with type 1 diabetes mellitus. Drugs Aging. 2017;34:29-38.

25. Shiramoto M, Nishida T, Hansen AK, Haahr H. Fast-acting insulin aspart in Japanese patients with type 1 diabetes: faster onset, higher early exposure and greater early glucose-lowering effect relative to insulin aspart. J Diabetes Investig. 2018;9:303-10.

26. Hövelmann U, Heise T, Nosek L, Sassenfeld B, Thomsen KMD, Haahr H. Pharmacokinetic properties of fast-acting insulin aspart administered in different subcutaneous injection regions. Clin Drug Investig. 2017;37:503-9.

27. Novo Nordisk A/S. A trial investigating the pharmacokinetic properties of formulations of NN1218 in subjects with type 1 diabetes. ClinicalTrials.gov identifier: NCT01469143. https://clinicaltrials. gov/ct2/show/NCT01469143. Accessed 3 Oct 2019.

28. Mathieu C, Bode BW, Franek E, et al. Efficacy and safety of fastacting insulin aspart in comparison with insulin aspart in type 1 diabetes (onset 1): a 52-week, randomized, treat-to-target, phase III trial. Diabetes Obes Metab. 2018;20:1148-55.

29. Klonoff DC, Evans ML, Lane W, et al. A randomized, multicentre trial evaluating the efficacy and safety of fast-acting insulin aspart in continuous subcutaneous insulin infusion in adults with type 1 diabetes (onset 5). Diabetes Obes Metab. 2019;21:961-7.

30. Russell-Jones D, Bode BW, De Block C, et al. Fast-acting insulin aspart improves glycemic control in basal-bolus treatment for type 1 diabetes: Results of a 26-week multicenter, active-controlled, treat-to-target, randomized, parallel-group trial (onset 1). Diabetes Care. 2017;40:943-50.

31. Buse JB, Carlson AL, Komatsu M, et al. Fast-acting insulin aspart versus insulin aspart in the setting of insulin degludec-treated type 1 diabetes: efficacy and safety from a randomized double-blind trial. Diabetes Obes Metab. 2018;20:2885-93. 
32. Bowering K, Case C, Harvey J, et al. Faster aspart versus insulin aspart as part of a basal-bolus regimen in inadequately controlled type 2 diabetes: the onset 2 trial. Diabetes Care. 2017;40:951-7.

33. Lane W, Bozkurt K, Favaro E, et al. Efficacy and safety of fastacting insulin aspart compared with insulin aspart, both with insulin degludec with or without metformin, in adults with type 2 diabetes. Diabetologia. 2019;62(suppl. 1):S10-1.

34. Rodbard HW, Tripathy D, Vidrio Velázquez M, Demissie M, Tamer SC, Piletič M. Adding fast-acting insulin aspart to basal insulin significantly improved glycaemic control in patients with type 2 diabetes: a randomized, 18-week, open-label, phase 3 trial (onset 3). Diabetes Obes Metab. 2017;19:1389-96.

35. Bode BW, Iotova V, Kovarenko M, et al. Efficacy and safety of fast-acting insulin aspart compared with insulin aspart, both in combination with insulin degludec, in children and adolescents with type 1 diabetes: the onset 7 trial. Diabetes Care. 2019;42:1255-62.

36. US FDA. Inactive ingredient search for approved drug products. Available at: http://www.accessdata.fda.gov/scripts/cder/ iig/index.cfm. Accessed 3 Oct 2019.

37. US FDA. Select committee on GRAS substances database. Available at: https://www.accessdata.fda.gov/scripts/ $\mathrm{fdcc} /$ ?set=SCOGS. Accessed 3 Oct 2019.

38. Knip M, Douek IF, Moore WP, et al. Safety of high-dose nicotinamide: a review. Diabetologia. 2000;43:1337-45.

39. McNeal CJ, Meininger CJ, Reddy D, Wilborn CD, Wu G. Safety and effectiveness of arginine in adults. J Nutr. 2016;146:2587S-93S.

40. Brange J, Langkjaer L. Chemical stability of insulin. 3. Influence of excipients, formulation, and $\mathrm{pH}$. Acta Pharm Nord. 1992;4:149-58.

41. Březina K, Duboué-Dijon E, Palivec V, et al. Can arginine inhibit insulin aggregation? A combined protein crystallography, capillary electrophoresis, and molecular simulation study. J Phys Chem B. 2018;122:10069-76.

42. Zijlstra E, Demissie M, Graungaard T, Heise T, Nosek L, Bode B. Investigation of pump compatibility of fast-acting insulin aspart in subjects with type 1 diabetes. J Diabetes Sci Technol. 2018;12:145-51.

43. Bode BW, Johnson JA, Hyveled L, Tamer SC, Demissie M. Improved postprandial glycemic control with faster-acting insulin aspart in patients with type 1 diabetes using continuous subcutaneous insulin infusion. Diabetes Technol Ther. 2017;19:25-33.

44. Heise T, Zijlstra E, Nosek L, Heckermann S, Plum-Mörschel L, Forst T. Euglycaemic glucose clamp: what it can and cannot do, and how to do it. Diabetes Obes Metab. 2016;18:962-72.

45. Jain L, Parks MH, Sahajwalla C. Determination of time to onset and rate of action of insulin products: importance and new approaches. J Pharm Sci. 2013;102:271-9.

46. Steiner S, Hompesch M, Pohl R, et al. A novel insulin formulation with a more rapid onset of action. Diabetologia. 2008;51:1602-6.

47. Lindholm A, Jacobsen LV. Clinical pharmacokinetics and pharmacodynamics of insulin aspart. Clin Pharmacokinet. 2001;40:641-59.

48. Becker RH, Frick AD. Clinical pharmacokinetics and pharmacodynamics of insulin glulisine. Clin Pharmacokinet. 2008;47:7-20

49. Morrow L, Muchmore DB, Hompesch M, Ludington EA, Vaughn DE. Comparative pharmacokinetics and insulin action for three rapid-acting insulin analogs injected subcutaneously with and without hyaluronidase. Diabetes Care. 2013;36:273-5.

50. Arnqvist H, Olsson PO, von Schenck H. Free and total insulin as determined after precipitation with polyethylene glycol: analytical characteristics and effects of sample handling and storage. Clin Chem. 1987;33:93-6.

51. Sapin R. Anti-insulin antibodies in insulin immunometric assays: a still possible pitfall. Eur J Clin Chem Clin Biochem. 1997;35:365-7.

52. Heise T, Nosek L, Spitzer H, et al. Insulin glulisine: a faster onset of action compared with insulin lispro. Diabetes Obes Metab. 2007;9:746-53.

53. Arnolds S, Rave K, Hövelmann U, Fischer A, Sert-Langeron C, Heise T. Insulin glulisine has a faster onset of action compared with insulin aspart in healthy volunteers. Exp Clin Endocrinol Diabetes. 2010;118:662-4.

54. Swinnen SG, Holleman F, DeVries JH. The interpretation of glucose clamp studies of long-acting insulin analogues: from physiology to marketing and back. Diabetologia. 2008;51:1790-5.

55. Becker RH, Frick AD, Nosek L, Heinemann L, Rave K. Doseresponse relationship of insulin glulisine in subjects with type 1 diabetes. Diabetes Care. 2007;30:2506-7.

56. Freidenberg GR, Suter SL, Henry RR, Reichart D, Olefsky $\mathrm{JM}$. In vivo stimulation of the insulin receptor kinase in human skeletal muscle. Correlation with insulin-stimulated glucose disposal during euglycemic clamp studies. J Clin Invest. 1991;87:2222-9.

57. Heinemann L. Variability of insulin action: does it matter? Insulin. 2008;3:37-45.

58. Herring R, Jones RH, Russell-Jones DL. Hepatoselectivity and the evolution of insulin. Diabetes Obes Metab. 2014;16:1-8.

59. Heise T, Linnebjerg H, Cao D, et al. Ultra rapid lispro (URLi) lowers postprandial glucose (PPG) and more closely matches normal physiological glucose response compared with other rapid insulin analogs [poster 1112-P]. In: American Diabetes association 79th scientific sessions, 7-11 June 2019; San Francisco.

60. Pozzilli P, Battelino T, Danne T, Hovorka R, Jarosz-Chobot P, Renard E. Continuous subcutaneous insulin infusion in diabetes: patient populations, safety, efficacy, and pharmacoeconomics. Diabetes Metab Res Rev. 2016;32:21-39.

61. Paglialunga S, Offman E, Ichhpurani N, Marbury TC, Morimoto $\mathrm{BH}$. Update and trends on pharmacokinetic studies in patients with impaired renal function: practical insight into application of the FDA and EMA guidelines. Expert Rev Clin Pharmacol. 2017;10:273-83.

62. Verbeeck RK. Pharmacokinetics and dosage adjustment in patients with hepatic dysfunction. Eur J Clin Pharmacol. 2008;64:1147-61.

63. Holmes G, Galitz L, Hu P, Lyness W. Pharmacokinetics of insulin aspart in obesity, renal impairment, or hepatic impairment. Br J Clin Pharmacol. 2005;60:469-76.

64. Morello CM. Pharmacokinetics and pharmacodynamics of insulin analogs in special populations with type 2 diabetes mellitus. Int J Gen Med. 2011;4:827-35.

65. Frid AH, Kreugel G, Grassi G, et al. New insulin delivery recommendations. Mayo Clin Proc. 2016;91:1231-55.

66. ter Braak EW, Woodworth JR, Bianchi R, et al. Injection site effects on the pharmacokinetics and glucodynamics of insulin lispro and regular insulin. Diabetes Care. 1996;19:1437-40.

67. Mudaliar SR, Lindberg FA, Joyce M, et al. Insulin aspart (B28 asp-insulin): a fast-acting analog of human insulin: absorption kinetics and action profile compared with regular human insulin in healthy nondiabetic subjects. Diabetes Care. 1999;22:1501-6.

68. Heise T, Bott S, Tusek C, et al. The effect of insulin antibodies on the metabolic action of inhaled and subcutaneous insulin: a prospective randomized pharmacodynamic study. Diabetes Care. 2005;28:2161-9. 
69. Chen JW, Frystyk J, Lauritzen T, Christiansen JS. Impact of insulin antibodies on insulin aspart pharmacokinetics and pharmacodynamics after 12 -week treatment with multiple daily injections of biphasic insulin aspart 30 in patients with type 1 diabetes. Eur J Endocrinol. 2005;153:907-13.

70. Lindholm A, Jensen LB, Home PD, Raskin P, Boehm BO, Råstam J. Immune responses to insulin aspart and biphasic insulin aspart in people with type 1 and type 2 diabetes. Diabetes Care. 2002;25:876-82.
71. Cengiz E, Bode B, Van Name M, Tamborlane WV. Moving toward the ideal insulin for insulin pumps. Expert Rev Med Devices. 2016;13:57-69.

72. Majeed W, Thabit H. Closed-loop insulin delivery: current status of diabetes technologies and future prospects. Expert Rev Med Devices. 2018;15:579-90.

73. Evans M, Ceriello A, Danne T. Use of fast-acting insulin aspart in insulin pump therapy in clinical practice. Diabetes Obes Metab. 2019;21:2039-47. 